The Astrophysical Journal Supplement Series, 60:949-961, 1986 April

ic 1986. The American Astronomical Society. All rights reserved. Printed in U.S.A.

\title{
THE LABORATORY MILLIMETER-WAVE SPECTRUM OF METHYL FORMATE IN ITS GROUND TORSIONAL $E$ STATE
}

\author{
Grant M. Plummer, Eric Herbst, and Frank C. De Lucia \\ Department of Physics, Duke University \\ AND \\ Geoffrey A. BLAKE \\ Department of Chemistry, California Institute of Technology \\ Received 1985 September 25; accepted 1985 December 5
}

\begin{abstract}
Over 250 rotational transitions of the internal rotor methyl formate $\left(\mathrm{HCOOCH}_{3}\right)$ in its ground $\left(v_{t}=0\right)$ degenerate $(E)$ torsional substate have been measured in the millimeter-wave spectral region. These data and a number of $E$-state lines identified by several other workers have been analyzed using an extension of the classical principal-axis method in the high barrier limit. The resulting rotational constants allow accurate prediction of the $v_{t}=0 E$ substate methyl formate spectrum below $300 \mathrm{GHz}$ between states with angular momentum $J \leq 30$ and rotational energy $E_{\text {rot }} \leq 350 \mathrm{~cm}^{-1}$. The calculated transition frequencies for the $E$ state, when combined with the results of our previous analysis of the ground symmetric, nondegenerate $(A)$ state, account for over 200 of the emission lines observed toward Orion in a recent survey of the $215-265 \mathrm{GHz}$ band.
\end{abstract}

Subject headings: interstellar: molecules - laboratory spectra - line identifications molecular processes - transition probabilities

\section{INTRODUCTION}

Identification of the molecular species responsible for the growing number of millimeter and submillimeter emission lines observed toward astronomical sources is crucial to our understanding of the interstellar medium. Many of the observed features have been assigned to a relatively small number of molecular species; in recent millimeter-wave studies of Orion performed by Johansson et al. (1984), Sutton et al. (1985), and Blake et al. (1986), a substantial fraction of such features was found to be due to three molecules: methyl formate, dimethyl ether, and methanol. In part, the prevalence of these species in the Orion spectrum is due to their common feature of internal rotation, which results in a large number of observable rotational transitions for each, especially if the source is a warm one.

Since the identification of less prevalent species via their often sparse rotational spectra is of vital interest, complete descriptions of the rather complicated spectra of these known constituents of the intersteller medium must be made. Some progress in measuring and understanding the spectra of interstellar internal rotors has been made (Lovas, Lutz, and Dreizler 1979; Bauder 1979; Herbst et al. 1984). However, internal rotation greatly increases the difficulty of accurately calculating the transition frequencies of molecules. Many observable transitions involve levels which are strongly affected by the rigid-body rotation-internal rotation interactions, and whose energies are difficult to determine to within the frequency resolution of current astronomical receivers. As the sensitivity of these receivers increases, large numbers of such transitions will have to be observed in the laboratory or reliably predicted if the identification of the less abundant species is to be accomplished.

We have previously provided an accurate list of the 2700 strongest transitions below $500 \mathrm{GHz}$ of methyl formate in its ground torsional $A$ (symmetric) state involving rotational states with angular momentum up to $J=50$ and rotational energy below $350 \mathrm{~cm}^{-1}$ (Plummer et al. 1984). In this paper we present the laboratory millimeter-wave spectrum (up to $300 \mathrm{GHz}$ ) of methyl formate in its ground torsional $E$ (degenerate) state, including those states with angular momentum $J \leq 30$ and $E_{\text {rot }} \leq 350 \mathrm{~cm}^{-1}$. Other measurements of the methyl formate rotational spectrum have been made by Curl (1959) in the 8-30 GHz band, by Bauder (1979) in the range up to $58 \mathrm{GHz}$, and by DeMaison et al. (1984) in the 220-240 $\mathrm{GHz}$ band; we have made extensive use of these authors' observations and assignments. Over 250 new $E$-state transitions have been measured in our laboratory, and an analysis of a combined data set of $364 E$-state lines has been carried out. Table 1 lists the observed transitions, the calculated perturbations $(P)$ due to the rotational-internal rotation interaction (see below), and the residuals $(R)$ obtained in the fit. Our analyses differ from those of previous workers in that we handle the $A$ and $E$ states separately (rather than carrying out a global fit to the entire spectrum), and place emphasis on the accurate prediction of the ground-state rotational spectrum (rather than on the determination of molecular structural parameters). This approach has allowed greatly increased accuracy in fitting and in predicting the spectra, and is attractive also because most of the calculations required in the case of methyl formate can be carried out with existing principal-axis 
TABLE 1

Transitions USED IN the ANALYSIS

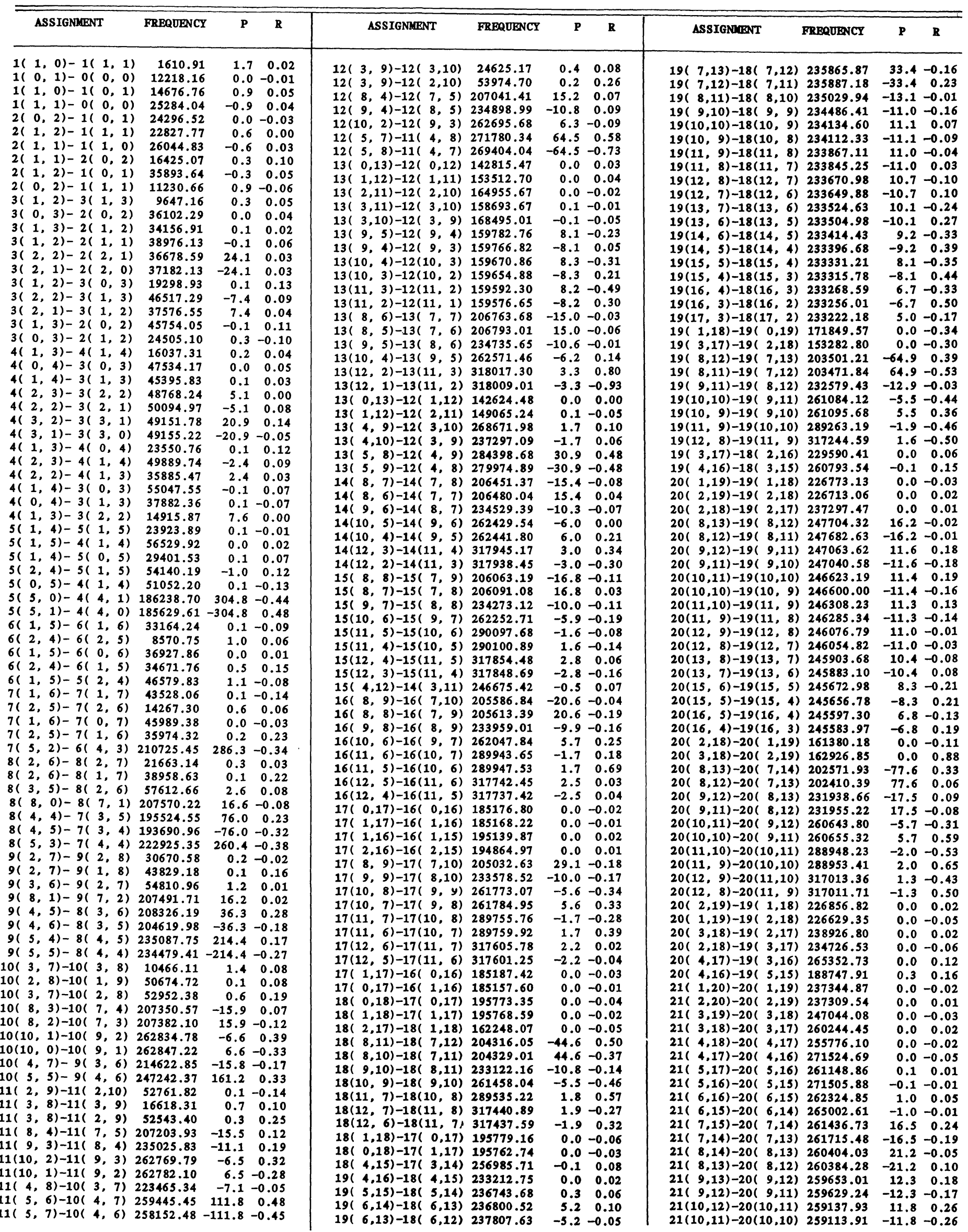


TABLE $1-$ Continued

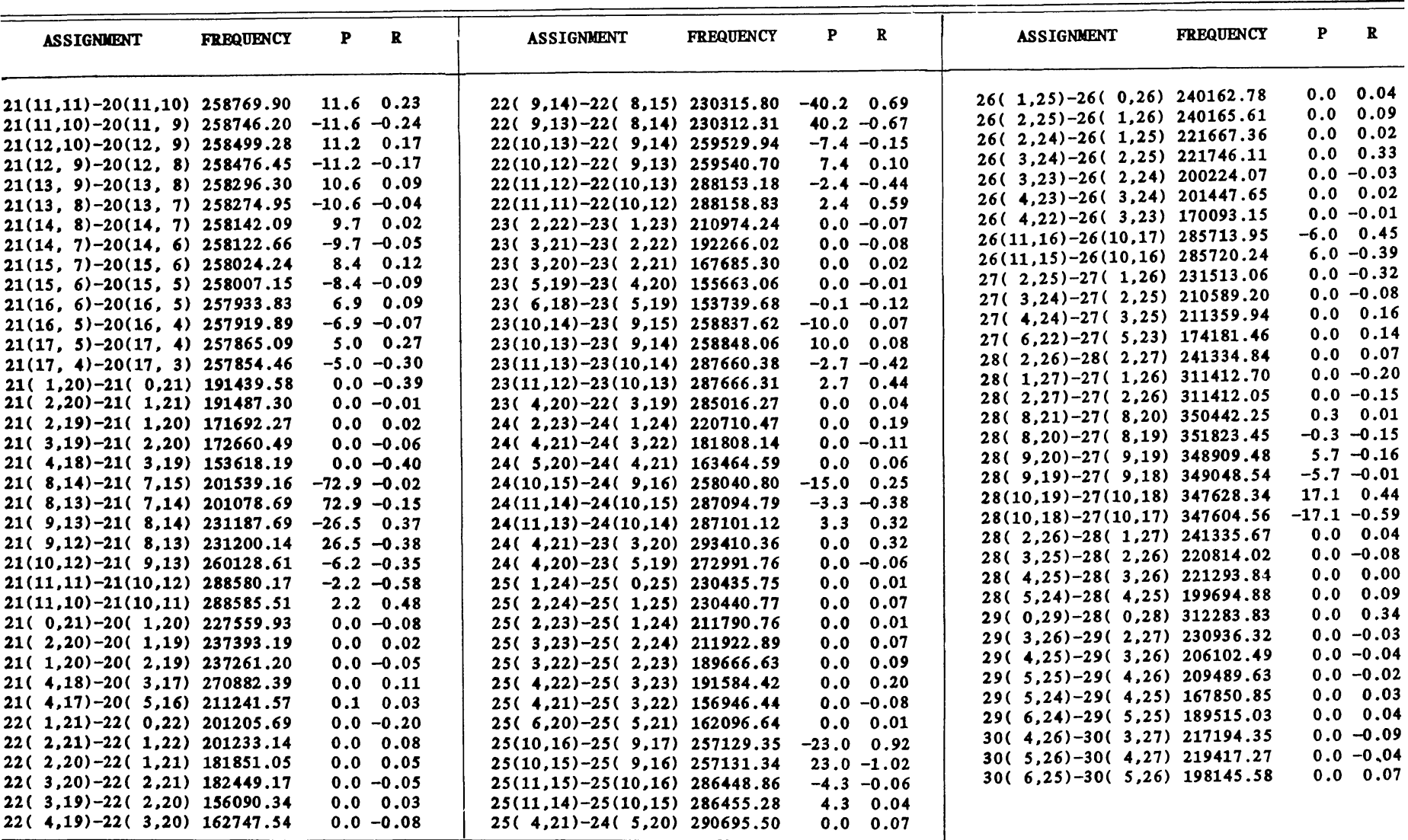

NOTE. - Transition frequencies and residuals ( $R$, observed minus calculated) in $\mathrm{MHz}$. Calculated perturbation of the pseudorigid rotor frequencies due to internal rotation $(P)$ in $\mathrm{MHz}$.

system asymmetric rotor fitting routines. Diagonalization of extended rotational Hamiltonian matrices, as undertaken by Herbst et al. (1984), is not required.

\section{EXPERIMENT AND THEORY}

Measurements of the methyl formate spectrum have been carried out with our standard millimeter/submillimeter wave spectrometer. Briefly, a $40-60 \mathrm{GHz}$ klystron is used to drive a waveguide-mounted silicon point-contact diode harmonic generator, which produces usable intensities of microwave power at multiples of the source frequency up to about $1 \mathrm{THz}$. After passing through a $2 \mathrm{~m}$ absorption cell containing $\sim 10 \mathrm{mTorr}$ of the sample, the microwave radiation is detected by a $1.4 \mathrm{~K}$ InSb chip. As the source frequency is swept, the response of the chip is monitored, stored in a digital averager, and transferred to a computer for measurement and analysis. Details of our experimental techniques can be found in the literature (De Lucia 1976; Helminger, Messer, and De Lucia 1983).

The effective Hamiltonian used in our analysis of the acquired data has been discussed fully by Herschbach (1957, 1959). The standard principal-axis method (PAM) Hamiltonian for internal rotors was subjected to a Van Vleck transformation through ninth order using modern computational techniques. In the limit of a high potential barrier to the torsional motion of the methyl group, this transformation allows the approximate separation of the $E$ and $A$ problems. For a given torsional state $v$ and symmetry $\sigma(\sigma=0$ for the $A$ state and $\sigma=1,-1$ for the $E$ states), the resulting effective Hamiltonian through ninth order is (Herschbach 1957, 1959)

$$
\boldsymbol{H}_{v \sigma}=\boldsymbol{H}_{\mathrm{rot}}+\boldsymbol{H}_{\mathrm{dist}}+\boldsymbol{H}_{\mathrm{tr}},
$$

TABLE 2

EFFECTIVE CONSTANTS of $v_{t}=0(E) \mathrm{HCOOCH}_{3}{ }^{a}$

\begin{tabular}{|c|c|c|c|}
\hline Constant & Value & Constant & Value \\
\hline & $19980.3907(105)$ & $L_{K K J} \times 10^{9}$ & $2.62(62)$ \\
\hline & $6913.6822(23)$ & $F \times 10^{-3} \ldots$ & $168.142(138)$ \\
\hline & $5304.5114(21)$ & & $31.8288(63)$ \\
\hline$\Delta_{J} \times 10^{4} \ldots$ & $58.895(25)$ & $\rho_{a} \times 10^{3}$. & $78.4580(945)$ \\
\hline$\Delta_{J K} \times 10^{4}$ & $-233.13(57)$ & $\delta_{F} \times 10^{2}$. & $-5.915(178)$ \\
\hline$\Delta_{K} \times 10^{4}$. & $754.009(749)$ & $\delta_{\rho} \times 10^{6} \ldots$ & $-7.333(655)$ \\
\hline$\delta_{J} \times 10^{4}$ & $18.571(7)$ & $W_{01}^{(1)} \times 10^{3}$ & -2.85136 \\
\hline$\delta_{K} \times 10^{4} .$. & $20.57(52)$ & $W_{01}^{(3)} \times 10^{3}$ & 2.08631 \\
\hline$H_{J K} \times 10^{7}$ & $-9.770(920)$ & $W_{01}^{(5)} \times 10^{4}$ & -4.5909 \\
\hline$H_{K J} \times 10^{7}$ & $-34.11(46)$ & $W_{01}^{(7)} \times 10^{5}$ & 4.8583 \\
\hline$h_{J K} \times 10^{7}$. & $-3.35(74)$ & $W_{01}^{(9)} \times 10^{6}$ & -2.959 \\
\hline $\begin{array}{l}h_{K} \times 10 \\
L_{J K} \times 10^{9} .\end{array}$ & $\begin{array}{r}-6.407(59) \\
1.07(46)\end{array}$ & & \\
\hline
\end{tabular}

NorE.-Number of observed transitions: 364 ; variance of fit: 0.25 $\mathrm{MHz}$. Numbers in parentheses are $1 \sigma$ deviations.

${ }^{a}$ Number of significant digits needed to reproduce spectrum. Rotational constants and centrifugal distortion constants from $A$ through $F$ and $\delta_{F}$ are in $\mathrm{MHz}$. All other parameters $\left(s, \rho_{a}, \delta_{\rho}\right.$, and the $\left.W_{01}\right)$ are dimensionless. 
TABLE 3

TRANSITIONS FROM 1 TO $300 \mathrm{GHz}$

\begin{tabular}{|c|c|c|c|c|c|c|c|c|c|c|c|c|c|c|c|c|c|c|}
\hline \multicolumn{3}{|c|}{ ASSIGNMENT } & \multicolumn{2}{|c|}{ FREQUENCY } & $\mathbf{s}$ & $\mathrm{E}$ & \multicolumn{2}{|r|}{ ASSIGNMENT } & \multicolumn{2}{|c|}{ FREQUENCY } & $\mathbf{s}$ & E & \multicolumn{2}{|r|}{ ASSIGNOENT } & \multicolumn{2}{|c|}{ FREQUENCY } & $\mathbf{8}$ & B \\
\hline 30( & $9,21)-30($ & $9,22)$ & 1038.43 & $(0.02)$ & 4.7 & 229 & 251 & $6,19)-25(6,20)$ & 34476.02 & $(0.14)$ & 1.8 & 152 & 141 & $4,10)-14(3,11)$ & 71988.46 & $(0.03)$ & 10.4 & 51 \\
\hline 26( & $8,18)-26($ & $8,19)$ & 1138.32 & $(0.03)$ & 4.3 & 174 & 61 & $2,4)-6(1,5)$ & 34671.59 & $(0.02)$ & 5.1 & 11 & & & 72680.83 & $(0.02)$ & 5.3 & 10 \\
\hline 14( & $5,9)-14($ & $5,10)$ & 1171.72 & $(0.03)$ & 3.2 & 55 & 211 & $5,16)-21(5,17)$ & 35854.29 & $(0.10)$ & 1.5 & 108 & 68 & $0,5)$ & 73033.20 & $(0.02)$ & 4.4 & 8 \\
\hline 221 & $7,15)-22($ & $7,16)$ & 1211.83 & $(0.05)$ & 3.9 & 127 & 71 & $2,5)-7(1,6)$ & 35974.08 & $(0.02)$ & 6.1 & 14 & 61 & $5,1)-5(5,0)$ & 73658.27 & $(0.02)$ & 1.8 & 20 \\
\hline 18( & $6,12)-18($ & $6,13)$ & 1233.45 & $(0.04)$ & 3.6 & 87 & 171 & $4,13)-17(4,14)$ & 35978.61 & $(0.08)$ & 1.2 & 72 & 61 & $5,2)-5(5,1)$ & 73663.99 & $(0.02)$ & 1.8 & 20 \\
\hline & & $3,5)$ & 1528.73 & $(0.01)$ & 2.3 & 16 & 31 & $0,3)-2(0,2)$ & 36102.25 & $(0.01)$ & 3.0 & 2 & 61 & $(4,2)-5(4,1)$ & 73783.00 & $(0.02)$ & 3.3 & 16 \\
\hline $1 i$ & $1,0)-1($ & $1,1)$ & 1610.89 & $(0.00)$ & 1.5 & 1 & 31 & $2,2)-2(2,1)$ & 36678.57 & $(0.02)$ & 1.7 & 4 & 61 & $(4,3)-5(4,2)$ & 73787.60 & $(0.02)$ & 3.3 & 16 \\
\hline $27 i$ & $8,19)-27($ & $8,20)$ & 1986.88 & $(0.03)$ & 4.0 & 186 & 3( & $2,1)-2(2,0)$ & 37182.10 & $(0.02)$ & 1.7 & 4 & 61 & $3,4)-5(3,3)$ & 73905.90 & $(0.03)$ & 4.5 & 13 \\
\hline 11( & $4,7)-11($ & $4,8)$ & 2003.24 & $(0.01)$ & 2.6 & 35 & 81 & $2,6)-8(1,7)$ & 38958.41 & $(0.02)$ & 6.7 & 17 & & $3,3)-5(3,2)$ & 74263.48 & $(0.03)$ & 4.5 & 13 \\
\hline 4( & & $2,3)$ & 2033.07 & $(0.01)$ & 1.7 & 6 & 31 & $1,2)-2(1,1)$ & 38976.08 & $(0.01)$ & 2.7 & 3 & 131 & $(4,9)-13(3,10)$ & 75551.69 & $(0.03)$ & 9.0 & 45 \\
\hline $23 i$ & $7,16)-23($ & $7,17)$ & 2149.26 & $(0.03)$ & 3.7 & 137 & $9 i$ & $2,7)-9(1,8)$ & 43829.02 & $(0.03)$ & 7.0 & 21 & 151 & $3,13)-16(0,16)$ & 75552.64 & $(0.11)$ & 0.0 & 53 \\
\hline $15 i$ & $5,10)-15($ & $5,11)$ & 2213.28 & $(0.02)$ & 2.9 & 61 & 30( & $7,23)-30(7,24)$ & 44333.72 & $(0.33)$ & 2.0 & 217 & 18( & $4,14)-18(3,15)$ & 75652.72 & $(0.05)$ & 14.1 & 79 \\
\hline $19 i$ & $6,13)-19($ & $6,14)$ & 2240.70 & $(0.03)$ & 3.3 & 95 & 41 & $1,4)-3(1,3)$ & 45395.80 & $(0.02)$ & 3.7 & 4 & & & 76018.17 & $(0.03)$ & 5.2 & 11 \\
\hline 81 & & $3,6)$ & 3216.48 & $(0.01)$ & 2.0 & 19 & $14 i$ & $3,11)-14(3,12)$ & 45847.38 & $(0.07)$ & 0.8 & 49 & 20 & $4,16)-20(4,17)$ & 76670.59 & $(0.11)$ & 0.9 & 96 \\
\hline $28 i$ & $8,20)-28($ & $8,21)$ & 3368.24 & $(0.04)$ & 3.8 & 197 & 261 & $6,20)-26(6,21)$ & 46920.02 & $(0.17)$ & 1.7 & 164 & 61 & $2,4)-5(2,3)$ & 76701.82 & $(0.02)$ & 5.3 & 11 \\
\hline 24( & $7,17)-24($ & $7,18)$ & 3694.07 & $(0.03)$ & 3.4 & 147 & 41 & $0,4)-3(0,3)$ & 47534.12 & $(0.02)$ & 4.0 & 4 & $23(1$ & $14,9)-24(13,12)$ & 76794.92 & $(0.57)$ & 1.2 & 203 \\
\hline 12( & $4,8)-12($ & $4,9)$ & 3806.25 & $(0.01)$ & 2.3 & 40 & 18( & $4,14)-18(4,15)$ & 48072.09 & $(0.09)$ & 1.1 & 79 & 6( & $1,5)-5(1,4)$ & 76796.09 & $(0.02)$ & 5.8 & 10 \\
\hline 201 & $6,14)-20($ & $6,15)$ & 3927.19 & $(0.03)$ & 3.0 & 103 & 221 & $5,17)-22(5,18)$ & 48291.36 & $(0.12)$ & 1.4 & 118 & 281 & $6,22)-28(6,23)$ & 77870.92 & $(0.27)$ & 1.4 & 188 \\
\hline 16( & $5,11)-16($ & $5,12)$ & 3997.30 & $(0.02)$ & 2.7 & 68 & 41 & $2,3)-3(2,2)$ & 48768.25 & $(0.02)$ & 3.0 & 6 & 241 & $(5,19)-24(5,20)$ & 78377.44 & $(0.15)$ & 1.2 & 138 \\
\hline 17( & $7,11)-16($ & $(8,8)$ & 4548.55 & $(0.23)$ & 1.4 & 85 & 4( & $3,2)-3(3,1)$ & 49151.64 & $(0.02)$ & 1.8 & 8 & 211 & $4,18)-22(2,21)$ & 78377.47 & $(0.09)$ & 0.0 & 102 \\
\hline $5 i$ & $2,3)-5($ & $2,4)$ & 4549.71 & $(0.01)$ & 1.4 & 8 & 41 & $3,1)-3(3,0)$ & 49155.27 & $(0.02)$ & 1.8 & 8 & 71 & $(1,7)-6(1,6)$ & 78479.38 & $(0.03)$ & 6.8 & 11 \\
\hline $2 i$ & $1,1)-2($ & $(1,2)$ & 4827.93 & $(0.00)$ & 0.8 & 2 & 41 & $2,2)-3(2,1)$ & 50094.90 & $(0.02)$ & 3.0 & 6 & & & 79401.70 & $(0.03)$ & 4.6 & 23 \\
\hline 291 & $8,21)-29($ & $8,22)$ & 5538.50 & $(0.07)$ & 3.5 & 210 & 10( & $2,8)-10(1,9)$ & 50674.63 & $(0.04)$ & 6.9 & 25 & 71 & $0,7)-6(0,6)$ & 79781.68 & $(0.03)$ & 6.9 & 11 \\
\hline 91 & $3,6)-9($ & $3,7)$ & 6079.82 & $(0.02)$ & 1.7 & 23 & 41 & $1,3)-3(1,2)$ & 51785.97 & $(0.02)$ & 3.7 & 5 & 121 & $8)-12(3,9)$ & 79840.81 & $(0.04)$ & 7.7 & 40 \\
\hline $25 i$ & $7,18)-25 i$ & $7,19)$ & 6131.78 & $(0.05)$ & 3.2 & 157 & 11( & $3,8)-11(2,9)$ & 52543.12 & $(0.02)$ & 9.0 & 32 & $13 i$ & $2,11)-13(1,12)$ & 81167.92 & $(0.06)$ & 6.3 & 41 \\
\hline 21( & $6,15)-21($ & $6,16)$ & 6605.01 & $(0.04)$ & 2.8 & 112 & 10( & $3,7)-10(2,8)$ & 52952.16 & $(0.02)$ & 7.9 & 27 & 161 & $3,13)-16(2,14)$ & 81314.20 & $(0.07)$ & 10.0 & 62 \\
\hline $13 i$ & $4,9)-13($ & $4,10)$ & 6749.78 & $(0.02)$ & 2.0 & 45 & 121 & $3,9)-12(2,10)$ & 53974.42 & $(0.02)$ & 9.9 & 37 & 71 & $1,7)-6(0,6)$ & 82242.89 & $(0.03)$ & 5.3 & 11 \\
\hline $17 i$ & $5,12)-17($ & $5,13)$ & 6858.04 & $(0.03)$ & 2.4 & 75 & & $3,6)-9(2,7)$ & 10.91 & $(0.03)$ & 6.6 & 23 & & $-19(3,16)$ & 82523.26 & $(0.07)$ & 14.0 & 88 \\
\hline & $2,4)-6($ & & 8570.69 & $(0.01)$ & 1.1 & 11 & 51 & $1,5)-4(1,4)$ & 56529.90 & $(0.02)$ & 4.8 & 6 & 10( & $3,8)-10(2,9)$ & 83605.14 & $(0.03)$ & 5.0 & 27 \\
\hline 301 & $8,22)-30($ & $8,23)$ & $\mathbf{8 8 2 8 . 0 7}$ & $(0.12)$ & 3.3 & 222 & $13 i$ & $3,10)-13(2,11)$ & 57513.79 & $(0.03)$ & 10.4 & 43 & 111 & 4.,7)-11( 3,8$)$ & 84224.57 & $(0.04)$ & 6.6 & 35 \\
\hline $26 i$ & $7,19)-26($ & $7,20)$ & 9819.00 & $(0.07)$ & 2.9 & 168 & 81 & $3,5)-8(2,6)$ & 57612.55 & $(0.03)$ & 5.4 & 19 & 71 & $2,6)-6(2,5)$ & 84449.17 & $(0.02)$ & 6.4 & 13 \\
\hline 10( & & $3,8)$ & 10466.03 & $(0.02)$ & 1.4 & 27 & $5 i$ & $0,5)-4(0,4)$ & 58565.70 & $(0.02)$ & 4.9 & 6 & 211 & $-21(4,17)$ & 85761.59 & $(0.05)$ & 16.9 & 108 \\
\hline 221 & $6,16)-22($ & $6,17)$ & 10647.70 & $(0.05)$ & 2.5 & 122 & $15 i$ & $3,12)-15(3,13)$ & 58626.18 & $(0.08)$ & 0.8 & 55 & 201 & $-20(4,16)$ & 85780.44 & $(0.05)$ & 15.8 & 99 \\
\hline 18( & $5,13)-18($ & $5,14)$ & 11173.78 & $(0.04)$ & 2.2 & 83 & $11 i$ & $2,9)-11(1,10)$ & 59405.79 & $(0.04)$ & 6.7 & 30 & & $-6(6,0)$ & 85919.15 & $(0.03)$ & 1.9 & 28 \\
\hline 14( & $4,10)-14($ & $4,11)$ & 11203.03 & $(0.03)$ & 1.8 & 51 & 51 & $2,4)-4(2,3)$ & 60780.32 & $(0.02)$ & 4.2 & 8 & 71 & $6,2)-6$ & 85926.66 & $(0.03)$ & 1.9 & 28 \\
\hline 11 & $0,1)-0($ & $0,0)$ & 12218.17 & $(0.00)$ & 1.0 & 0 & $5 i$ & $-4(4,0)$ & 61401.05 & $(0.02)$ & 1.8 & 14 & 71 & & & $(0.03)$ & 3.4 & 23 \\
\hline 71 & $2,5)-7($ & $2,6)$ & 14267.25 & $(0.02)$ & 0.9 & 14 & $5 i$ & $4,2)-4(4,1)$ & 61405.12 & $(0.02)$ & 1.8 & 14 & & & 86027.80 & $(0.03)$ & 3.4 & 23 \\
\hline $27 i$ & $7,20)-27($ & $7,21)$ & 15154.48 & $(0.11)$ & 2.7 & 180 & $27 i$ & $6,21)-27(6,22)$ & 61488.10 & $(0.22)$ & 1.5 & 175 & & & 86223.61 & $(0.03)$ & 4.7 & 19 \\
\hline $23 i$ & $6,17)-23($ & $6,18)$ & 16443.42 & $(0.08)$ & 2.3 & 131 & $5 i$ & $3,3)-4(3,2)$ & 61535.11 & $(0.02)$ & 3.2 & 10 & & & & $(0.03)$ & 4.7 & 19 \\
\hline 111 & $3,8)-11($ & $3,9)$ & 16618.22 & $(0.0$ & 1.2 & 32 & 51 & $4(3$, & & (0. & 3.2 & 10 & & & 3.73 & & 5.7 & 16 \\
\hline 19( & $5,14)-19($ & $5,15)$ & 17301.67 & $(0.06)$ & 1.9 & 91 & 19( & $4,15)-19(4,16)$ & 61750.98 & $(0.10)$ & 1.0 & 88 & & & 87143.29 & $(0.03)$ & 5.7 & 16 \\
\hline $15 i$ & $4,11)-15($ & $4,12)$ & 17472.96 & $(0.05)$ & 1.6 & 57 & 23( & $5,18)-23(5,19)$ & 62578.90 & $(0.14)$ & 1.2 & 128 & 81 & $7(1$. & 87766.42 & $(0.03)$ & 6.2 & 14 \\
\hline & & $2,7)$ & 2166 & 10.0 & 0.8 & 17 & & $2,3)-4(2,2)$ & 63296.96 & $(0.02)$ & 4.2 & 8 & 191 & $5,14)-19(4,15)$ & 88053.80 & $(0.05)$ & 14.3 & 91 \\
\hline 281 & $7,21)-28($ & $7,22)$ & 22520.65 & $(0.16)$ & 2.4 & 192 & 14( & $3,11)-14(2,12)$ & 63300.34 & $(0.04)$ & 10.5 & 49 & 100 & 4 & & $(0.04)$ & 5.6 & 30 \\
\hline & $1,2)-1($ & $1,1)$ & 22827.77 & $(0.01)$ & 1.5 & 2 & $5 i$ & $1,4)-4(1,3)$ & 64416.52 & $(0.02)$ & 4.8 & 7 & 221 & $5,17)-22(4,18)$ & 88337.51 & $(0.07)$ & 17.5 & 118 \\
\hline 21 & $0,2)-1($ & $0,1)$ & 24296.55 & $(0.01)$ & 2.0 & 1 & 61 & $1,6)-5(1,5)$ & 67555.64 & $(0.02)$ & 5.8 & 8 & 111 & $3,9)-11(2,10)$ & 88686.87 & $(0.03)$ & 5.3 & 31 \\
\hline 241 & $6,18)-24($ & $6,19)$ & 24321.54 & $(0.10)$ & 2.1 & 142 & 61 & $0,6)-5(0,5)$ & 69269.69 & $(0.02)$ & 5.9 & 8 & & $1,6)-6(1,5)$ & 88843.24 & & 6.8 & 12 \\
\hline 121 & $3,9)-12($ & $3,10)$ & 24625.09 & $(0.05)$ & 1.1 & 37 & 241 & $8,17)-23(9,14)$ & 69270.69 & $(0.27)$ & 2.5 & 153 & & & & & 7.8 & 14 \\
\hline 201 & $5,15)-20($ & $5,16)$ & 25497.27 & $(0.08)$ & 1.7 & 99 & 16( & $4,12)-16(3,13)$ & 69417.75 & $(0.03)$ & 12.9 & 64 & & & 90145.72 & $(0.03)$ & 6.4 & 14 \\
\hline 161 & $4,12)-16($ & $4,13)$ & 25730.22 & $(0.06)$ & 1.4 & 64 & 41 & $3,2)-4(2,3)$ & 69723.64 & $(0.06)$ & 1.7 & 8 & 81 & $(0,8)-7(0,7)$ & 90227.63 & $(0.03)$ & 7.9 & 14 \\
\hline & & & & & 1.5 & & & & & & 6.5 & 35 & & & & & & 26 \\
\hline 291 & $7,22)-29($ & $7,23)$ & 32205.52 & $(0.23)$ & 2.2 & 204 & 15( & $4,11)-15(3,12)$ & 69771.04 & $(0.03)$ & 11.8 & 57 & 81 & $1,8)$ & 91775.86 & $(0.03)$ & 6.3 & 14 \\
\hline & & $1,2)$ & 34156.89 & $(0.01)$ & 2.7 & 3 & 17( & $4,13)-17(3,14)$ & 71303.75 & $(0.04)$ & 13.7 & 72 & 201 & $(4,16)-20(3,17)$ & 91776.73 & $(0.08)$ & 13.7 & 96 \\
\hline 131 & $3,10)-13($ & $3,11)$ & 34426.48 & $(0.06)$ & 0.9 & 43 & 15( & $3,12)-15(2,13)$ & 71309.84 & $(0.06)$ & 10.4 & 55 & 181 & $5,13)-18(4,14)$ & 92073.90 & $(0.05)$ & 12.8 & 83 \\
\hline
\end{tabular}


TABLE 3-Continued

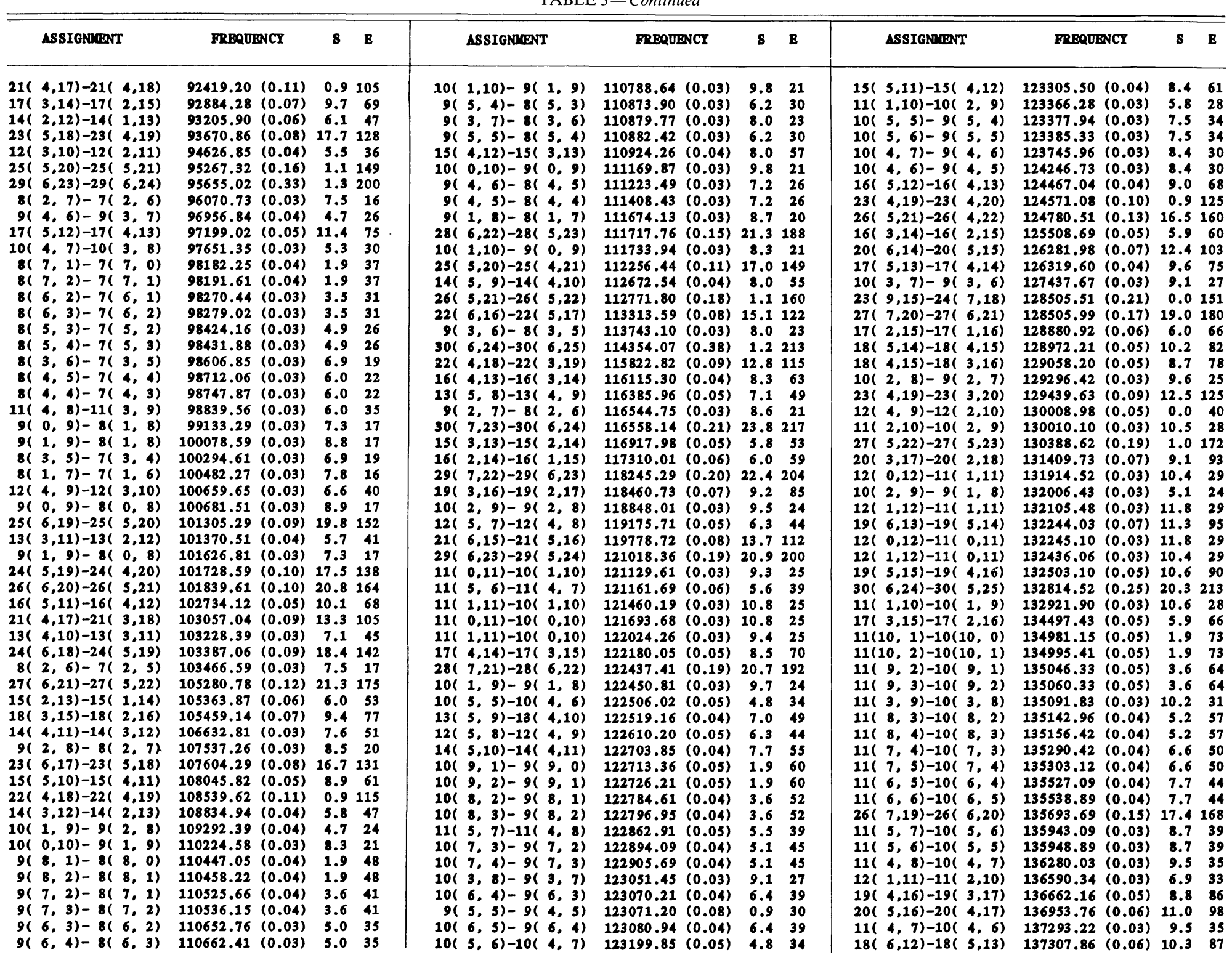


TABLE 3-Continued

\begin{tabular}{|c|c|c|c|c|c|c|c|c|c|c|c|c|c|c|}
\hline ASSIGNUENT & \multicolumn{2}{|c|}{ FRBQUENCY } & 8 & B & ASSIGNART & FREQUE & ENCY & $\mathbf{s}$ & B & ASSIGNUENT & \multicolumn{2}{|c|}{ FRERUENCT } & 8 & B \\
\hline $227-261908$ & & & & & & & & & & & & & & \\
\hline & 190017 & $(0.03)$ & & & $12(6,7)-12(5,8)$ & 150504.47 & $(0.05)$ & & & $20(3,18)-20(2,19)$ & 162925.96 & $.05)$ & 6. & \\
\hline $\begin{array}{l}18(2,16)-18(1,17) \\
24(20)-24(4,1)\end{array}$ & $\begin{array}{l}140047.36 \\
1.14013 .36\end{array}$ & $(0.10)$ & 6.0 & $\begin{array}{r}73 \\
135\end{array}$ & $12(4,8)-11(4,7)$ & $\begin{array}{l}150600.78 \\
15055\end{array}$ & $(0.03)$ & 10.7 & 40 & $24(5,20)-24(4.21)$ & 163464.51 & $(0.06)$ & 11.8 & 36 \\
\hline $\begin{array}{l}24(4,20)-24(4,21) \\
12(2,11)-11(2,10)\end{array}$ & $\begin{array}{l}140113.36 \\
141037.69\end{array}$ & $\begin{array}{l}(0.10) \\
(0.03)\end{array}$ & 0.9 & & $\begin{array}{l}19(2,17)-19(1,18) \\
22( \\
6\end{array}$ & $\begin{array}{l}150855.99 \\
150957.25\end{array}$ & $\begin{array}{l}(0.05) \\
(0.07)\end{array}$ & $\begin{array}{r}6.0 \\
12.8\end{array}$ & ${ }_{21}^{81}$ & $14(1,13)-13(1,12)$ & $\begin{array}{l}163829.61 \\
163925.81\end{array}$ & $\begin{array}{l}(0.03) \\
(0.03)\end{array}$ & 13.6 & 43 \\
\hline 8$)-10(3,7)$ & $\begin{array}{l}141244.02 \\
14124.02\end{array}$ & $(0.03)$ & $\begin{array}{l}11.6 \\
10.2\end{array}$ & & $11(6,6)-11(5,7)$ & $\begin{array}{l}151008.75 \\
15125\end{array}$ & $\begin{array}{l}(0.05) \\
(0.05)\end{array}$ & $\begin{array}{r}12.8 \\
4.9\end{array}-9$ & & $\begin{array}{l}15(0,15)-14(1,14) \\
15(1,15)-14(1,14)\end{array}$ & $\begin{array}{l}163925.81 \\
16396.35\end{array}$ & $\begin{array}{l}(0.03) \\
(0.03)\end{array}$ & $\begin{array}{l}13.4 \\
14.8\end{array}$ & 45 \\
\hline 11) $-17(5,12)$ & $\begin{array}{l}141354.23 \\
14163.05\end{array}$ & $(0.06)$ & 9.5 & 80 & $8(4,5)-8(2,6)$ & 151009.19 & $(0.08)$ & 0.0 & 22 & $15(0.15)-14(0.14)$ & 163987.43 & & & \\
\hline $11(2,9)-10(2,8)$ & $\begin{array}{l}141653.05 \\
14253\end{array}$ & 10.0 & 10.6 & & $11(6,5)-11(5,6)$ & $\begin{array}{l}151036 \\
151050\end{array}$ & & 4.9 & 44 & $15(1,15)-14(0,14)$ & 164021.97 & $(0.03)$ & 13.4 & 45 \\
\hline 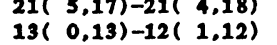 & $\begin{array}{l}142326.49 \\
142624.48\end{array}$ & $\begin{array}{l}(0.06) \\
(0.03)\end{array}$ & $\begin{array}{l}11.3 \\
11.4\end{array}$ & & $\begin{array}{l}13(2,12)-12(2,11) \\
14(2,12)-13(3,11)\end{array}$ & $\begin{array}{l}151950.04 \\
152780.28\end{array}$ & $\begin{array}{l}(0.03) \\
(0.05)\end{array}$ & $\begin{array}{r}12.6 \\
3.0\end{array}$ & $\begin{array}{l}38 \\
47\end{array}$ & $\begin{array}{l}29(5,24)-29(5,25) \\
13(4,9)-12(4,8)\end{array}$ & $\begin{array}{l}1641511.66 \\
164205.95\end{array}$ & $\begin{array}{l}(0.20) \\
(0.04)\end{array}$ & $\begin{array}{l}11.1 \\
11.8\end{array}$ & $\begin{array}{l}96 \\
45\end{array}$ \\
\hline $13(1,13)-12(1,12)$ & 142733.50 & $(0.03)$ & 12.8 & & $28(5.23)-28(4.24)$ & 153226.20 & $(0.16)$ & & & $13(2,11)-12(2,10)$ & 164955.70 & $(0.03)$ & & 41 \\
\hline $13(0,13)-12(0,12)$ & 142815.44 & $(0.03)$ & 12.8 & 34 & $19(3,17)-19(2,18)$ & & & 6.0 & 81 & $14(2.13)-13(1,12)$ & 165653.53 & $(0.03)$ & 9.1 & 43 \\
\hline $12(0,12)$ & 142924.46 & $(0.03)$ & 11.4 & & $14(0,14)-13(1,13)$ & & & 12.4 & & $-21(6,15)$ & & & & \\
\hline 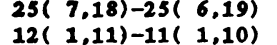 & $\begin{array}{l}143218.65 \\
143234.16\end{array}$ & $\begin{array}{l}\left(\begin{array}{l}(0.13) \\
(0.03)\end{array}\right) \\
\text { (a) }\end{array}$ & $\begin{array}{l}15.9 \\
11.6\end{array}$ & & $\begin{array}{ll}14(1,14)-13(1,13) \\
14(0.14)-13(0,13)\end{array}$ & $\begin{array}{l}153330.44 \\
153397.83\end{array}$ & & $\begin{array}{l}13.8 \\
13.8\end{array}$ & $\begin{array}{l}39 \\
39\end{array}$ & $24(5,20)-24(3,21)$ & 166654.25 & $(0.06)$ & & \\
\hline $\begin{array}{l}12(1,11)-11(1,10) \\
24(4,20)-24(3,21)\end{array}$ & $\begin{array}{l}143234.16 \\
14303.10\end{array}$ & $\begin{array}{l}(0.033) \\
(0.09)\end{array}$ & $\begin{array}{l}11.6 \\
12.3\end{array}$ & & 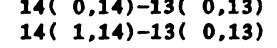 & $\begin{array}{l}153397.83 \\
153459.45\end{array}$ & $\begin{array}{l}(0.03) \\
(0.03)\end{array}$ & $\begin{array}{l}13.8 \\
12.4\end{array}$ & $\begin{array}{l}39 \\
39\end{array}$ & $\begin{array}{l}29(8,21)-29(7,22) \\
23(3,20)-23(2,21)\end{array}$ & $\begin{array}{l}167392.57 \\
167685.27\end{array}$ & $\begin{array}{l}(0.26) \\
(0.05)\end{array}$ & $\begin{array}{r}18.2 \\
9.0\end{array}$ & \\
\hline $18(3,16)-18(2,17)$ & 143784.05 & $(0.05)$ & 5.9 & 74 & $13(1,12)-12(1,11)$ & 153512.66 & $(0.03)$ & 12.6 & 38 & $26(6,21)-26(5,22)$ & 167691.39 । & $(0.08)$ & 14.4 & \\
\hline $21(3,18)-21(2,19)$ & $\begin{array}{l}143997.30 \\
14448.55\end{array}$ & $(0.06)$ & 9.0 & & $12(2,10)-11(2,9)$ & $\begin{array}{l}153553.25 \\
153618.57\end{array}$ & & 11.6 & $\begin{array}{r}35 \\
102\end{array}$ & $29(3,24)-29(4.25)$ & 167850.76 & $(0.17)$ & & \\
\hline $\begin{array}{l}\begin{array}{l}6,10)-16(6,11) \\
4,17)-20(3,18)\end{array} \\
4,18\end{array}$ & $\begin{array}{l}14448.53 \\
144886.55\end{array}$ & $\begin{array}{l}(0.07) \\
(0.05)\end{array}$ & $\begin{array}{l}8.7 \\
8.9\end{array}$ & & $\begin{array}{l}21(4,18)-21(3,19) \\
23(6,18)-23(5,19)\end{array}$ & $\begin{array}{l}153618.57 \\
153739.76\end{array}$ & $\begin{array}{l}(0.05) \\
(0.07)\end{array}$ & $\begin{array}{r}8.9 \\
13.3\end{array}$ & & $13(3,10)-12(3,9)$ & & & & \\
\hline 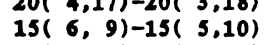 & $\begin{array}{l}146747.96 \\
146780\end{array}$ & $\begin{array}{l}(0.08) \\
(0.08)\end{array}$ & 7.9 & & $25(4,21)-25(4,22)$ & $\begin{array}{l}154891.70 \\
15470\end{array}$ & $\begin{array}{l}(0.09) \\
(0.07)\end{array}$ & $\begin{array}{l}13.3 \\
0.9\end{array}$ & $\begin{array}{l}131 \\
145\end{array}$ & $15(2,13)-14(3,12)$ & $\begin{array}{l}168788.36 \\
168914.76\end{array}$ & $\begin{array}{l}(0.08) \\
(0.04)\end{array}$ & & \\
\hline $12(3,10)-11(3,9)$ & 146977.67 & $(0.03)$ & 11.2 & 36 & $12(3,9)-11(3,8)$ & & & 11.3 & & $20(7,13)-20(6,14)$ & 169584.06 & & & \\
\hline $18(6,13)-18(5,14)$ & & & 10.1 & 87 & $23(5,19)-23(4,20)$ & $\begin{array}{l}155663.04 \\
15600.330\end{array}$ & & 11.7 & 126 & $26(4,22)-26(3,23)$ & 170093.13 & $(0.07)$ & & 156 \\
\hline $\begin{array}{l}12(11,1)-11(11,0) \\
12(11,2)-11(11,1)\end{array}$ & $\begin{array}{l}147750.42 \\
147265.76\end{array}$ & $\begin{array}{l}\left(\begin{array}{l}(0.05) \\
(0.05)\end{array}\right) \\
\text { (a) }\end{array}$ & $\begin{array}{l}1.9 \\
1.9\end{array}$ & 88 & $\begin{array}{l}\begin{array}{l}22(3,19)-2(2(2,20) \\
13(2,12)-12(1,1)\end{array}\end{array}$ & $\begin{array}{l}156090.30 \\
156397.39\end{array}$ & & $\begin{array}{l}9.0 \\
8.1\end{array}$ & $\begin{array}{r}111 \\
38\end{array}$ & $14(3,12)-13(3,11)$ & & & & \\
\hline $3(5,1)-6(3,4)$ & $\begin{array}{l}147266.59 \\
14203.10\end{array}$ & $(0.12)$ & 0.0 & $\begin{array}{l}88 \\
18\end{array}$ & $23(7,16)-23(6,17)$ & $\begin{array}{l}156758.64 \\
1564\end{array}$ & $(0.09)$ & $\begin{array}{r}8.1 \\
13.7\end{array}$ & & $23(7,17)-23(6,18)$ & $\begin{array}{l}171027.07 \\
171052.80\end{array}$ & $\begin{array}{l}(0.08) \\
(0.07)\end{array}$ & $\begin{array}{l}14.0 \\
13.3\end{array}$ & \\
\hline $19(6,14)-19(5,15)$ & 147305.00 & $(0.05)$ & 10.8 & 95 & $25(4,21)-25(3.22)$ & & & 12.2 & & $22(7,16)-22(6,17)$ & 171499.15 & & & \\
\hline $12(10,2)$ & & & 3.7 & 78 & $24(6,19)-$ & & & & & $5(6,20)$ & & & & \\
\hline $\begin{array}{l}12(10,3)-11(10,2) \\
12(9,3)-11(9,2)\end{array}$ & $\begin{array}{l}147325.73 \\
147396.92\end{array}$ & $\begin{array}{l}\left(\begin{array}{l}(0.05) \\
(0.05)\end{array}\right) \\
\end{array}$ & $\begin{array}{l}3.7 \\
5.3\end{array}$ & $\begin{array}{l}78 \\
69\end{array}$ & $\begin{aligned} 13(3,11)-12(3,10) \\
30(88,22)-30(7,23)\end{aligned}$ & $\begin{array}{l}158693 \\
15911\end{array}$ & $\begin{array}{l}(0.03) \\
(0.33)\end{array}$ & 12.2 & $\begin{array}{r}41 \\
222\end{array}$ & $(1,20)$ & 171692.25 & $(0.05)$ & & \\
\hline $12(9,4)-11(9,3)$ & $\begin{array}{l}147412.05 \\
14796\end{array}$ & $\begin{array}{l}(0.05) \\
(0.05)\end{array}$ & 3.3 & $\begin{array}{l}69 \\
69\end{array}$ & $\begin{array}{l}30(8,22)-30(7,23) \\
13(12,1)-12(12,0)\end{array}$ & $\begin{array}{l}15911 \\
15952\end{array}$ & & $\begin{array}{r}19.6 \\
1.9\end{array}$ & & $\begin{array}{l}14(13,1)-13(13,0) \\
14(13,2)-13(13,1)\end{array}$ & $\begin{array}{l}171793.63 \\
171809.74\end{array}$ & $\begin{array}{l}(0.07) \\
(0.07)\end{array}$ & $\begin{array}{l}1.9 \\
1.9\end{array}$ & ${ }_{121}^{121}$ \\
\hline $12(8,4)-11(8,3)$ & 147524.22 & $(0.04)$ & 6.7 & & $13(12,2)-12(12,1)$ & & & 1.9 & & $14(12,2)-13(12,1)$ & 171844.27 & & & \\
\hline $11(8,4)$ & $\begin{array}{l}147538.79 \\
10730.60\end{array}$ & $(0.04)$ & & & $13(11,2)-12(11,1)$ & & & 3.7 & 93 & $14(12,3$ & 171861.29 & $(0.05)$ & & \\
\hline $\begin{array}{l}17(6,12)-17(5,13) \\
2 \varepsilon(5,23)-28(5,24)\end{array}$ & $\begin{array}{l}147739.60 \\
147642.35\end{array}$ & $\left.\begin{array}{l}(0.06) \\
(0.19)\end{array}\right)$ & $\begin{array}{l}9.4 \\
1.0\end{array}-$ & & $\begin{array}{l}13(6,6) 8)-14(3,11) \\
13(11,3)-12(11,2)\end{array}$ & $\begin{array}{l}1595 \\
1595\end{array}$ & & 0.0 & $\begin{array}{l}54 \\
93\end{array}$ & $25(5,21)-25(4,22)$ & & & & \\
\hline $\begin{array}{l}28(3,23)-28(3,24) \\
1(2,11)-11(1,10)\end{array}$ & $\begin{array}{l}147642.35 \\
14681.51\end{array}$ & $\begin{array}{l}(0.19) \\
(0.03)\end{array}$ & $\begin{array}{l}1.0 \\
7.0\end{array}$ & & $\begin{array}{l}13(11,3)-12(11,2) \\
13(10,3)-12(10,2)\end{array}$ & $\begin{array}{l}139952.78 \\
159654.66\end{array}$ & & $\begin{array}{l}3.7 \\
5.3\end{array}$ & $\begin{array}{l}93 \\
83\end{array}$ & $14(11,3)-13(11,2)$ & $\begin{array}{l}171915.45 \\
171932.95\end{array}$ & $\begin{array}{l}(0.05) \\
(0.055)\end{array}$ & 5.4 & 99 \\
\hline $12(7,5)-11(7,4)$ & $\begin{array}{l}147717.65 \\
145\end{array}$ & $(0.04)$ & 7.9 & & $13(10,4)-12(10,3)$ & 159671 & & 5.3 & & $\begin{array}{l}14(10,4)-1 \\
14(10,-1\end{array}$ & & & 6.9 & 9 \\
\hline $12(7,6)-11(7,5)$ & 147731.43 & & 7.9 & & $13(9,4)-12(9,3)$ & & & 6.8 & 75 & $14(10,5)$ & & & & \\
\hline $\begin{array}{l}6,15)-20(5,16) \\
6,6)-11(6,5) \\
6\end{array}$ & $\begin{array}{l}147852.06 \\
14028.03\end{array}$ & $(0.06)$ & 11.5 & & $\begin{array}{l}13(9,5 \\
13(8,5 \\
\end{array}$ & & & 6.8 & $\begin{array}{l}75 \\
67\end{array}$ & & & & & \\
\hline 6,7$)-11(6,6)$ & $\begin{array}{l}148028.03 \\
148040.79\end{array}$ & $\begin{array}{l}(0.04) \\
(0.04)\end{array}$ & $\begin{array}{l}9.0 \\
9.0\end{array}$ & $\begin{array}{l}49 \\
49\end{array}$ & $\begin{array}{l}13(8,5 \\
13(8,6\end{array}$ & & & $\begin{array}{l}8.1 \\
8.1\end{array}$ & $\begin{array}{l}67 \\
67\end{array}$ & $\begin{array}{l}14(9,5)- \\
23(4,20)-\end{array}$ & .58 .828 & $\begin{array}{l}(0.05) \\
(0.04)\end{array}$ & 8.2 & 30 \\
\hline & $\begin{array}{l}148044.74 \\
14807\end{array}$ & $(0.07)$ & 8.6 & 73 & $13(7,6)-12(7,5)$ & & & 9.2 & 60 & $\begin{array}{l}(9,21) \\
(9,5)\end{array}$ & $1.81 \mathrm{C}$ & & $\begin{array}{l}9.0 \\
8.2\end{array}$ & \\
\hline ) & 148418.91 & $(0.6)-15$ & & & $13(7,7)-12(7,6)$ & & & & 60 & 2117 & & & 11.7 & \\
\hline $\begin{array}{r}\text { 8)-11( } 5,77) \\
18)-22(4,9)\end{array}$ & $\begin{array}{l}148545.06 \\
148585.77\end{array}$ & 0.0 & 9.5 & & $\begin{array}{l}23(5,19)-23(3,20) \\
13(6,7-12(6,6)\end{array}$ & 16053 & & 0.8 & & $14(8,6)-13$ & 172364.32 & & 9.4 & \\
\hline 7)-11( 5,6$)$ & $\begin{array}{l}148614.80 \\
148617\end{array}$ & $\begin{array}{l}(0.04) \\
(0.04)\end{array}$ & $\begin{array}{rl}11.6 & 9.9\end{array}$ & & $13(6,8)-12(6,7)$ & - & 10.012 & $\begin{array}{l}10.2 \\
10.2\end{array}$ & 54 & $\begin{array}{l}14(8,7)-13(8,6) \\
15(1,14)-14(2,13)\end{array}$ & $\begin{array}{l}172381.01 \\
172385.83\end{array}$ & & 10.1 & $\begin{array}{l}73 \\
49\end{array}$ \\
\hline $15(6,10)-15(5,11)$ & 148653.95 & & & & $14(1,13)-13(2,12)$ & 160944.89 & & 9.0 & & $1(2,20)$ & 172660.55 & & 6.0 & \\
\hline 8) & & & & & $13(5,9)-1$ & & & & & $13(7,6)$ & 172677.38 & & & \\
\hline 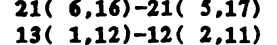 & $\begin{array}{l}149028.01 \\
14065.31\end{array}$ & & 12. & & $\begin{array}{l}13(4,10)-12(4,9) \\
20(2,18)-20\end{array}$ & $\begin{array}{l}161262.43 \\
16130.20\end{array}$ & & & $\begin{array}{l}45 \\
89\end{array}$ & - 8)-13( 7,7$)$ & 172693.27 & & & \\
\hline$\left(\begin{array}{l}1,12)-12(2,11) \\
(6,9)-14(5,10)\end{array}\right.$ & $\begin{array}{l}149296.46 \\
14063\end{array}$ & $\begin{array}{l}(0.03) \\
(0.07)\end{array}$ & $\begin{array}{l}8.4 \\
7 .\end{array}-1$. & 60 & $\begin{array}{l}20(2,18)-20(1,19) \\
13(5,8)-12(5,7)\end{array}$ & 161416.21 & & & & 200 & 172794.71 & & & \\
\hline & 149611.32 & $(0.06)$ & 6. & 54 & $22(7,15)-22(6,16)$ & 162063.29 & & & & $\begin{array}{l}6,8)-13(6,7) \\
6,8)\end{array}$ & $\begin{array}{l}173117.23 \\
173185.08\end{array}$ & & 11.0 & \\
\hline & 149924.42 & $(0.06)$ & & & $25(6,20)-25(5,21)$ & 162096.60 & & & & $4(6,9)-13(6,8)$ & 173194.40 & $(0.04)$ & & \\
\hline & & & & & & & & & & & & & & \\
\hline
\end{tabular}


TABLE 3-Continued

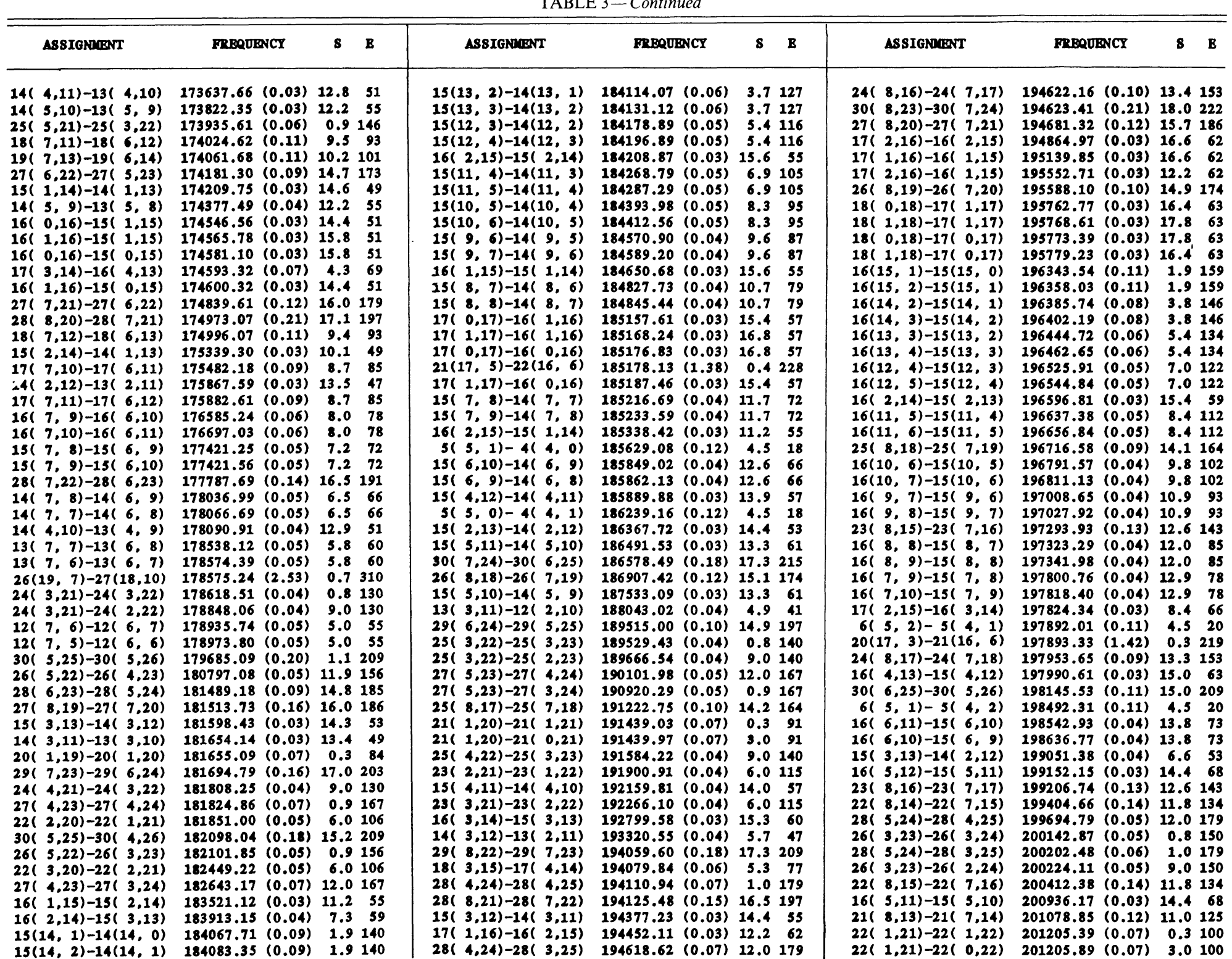


TABLE 3-Continued

\begin{tabular}{|c|c|c|c|c|c|c|c|c|c|c|c|c|c|c|c|c|c|}
\hline \multirow{2}{*}{\multicolumn{2}{|c|}{$\begin{array}{l}\text { ASSIGNGENT } \\
4,23)-26(3,24)\end{array}$}} & \multicolumn{2}{|c|}{ FREQUENCY } & & \multirow{2}{*}{$\begin{array}{c}\text { B } \\
150\end{array}$} & \multicolumn{3}{|c|}{ ASSIGNAENT } & \multicolumn{2}{|c|}{ FREQUENCY } & \multicolumn{2}{|r|}{ E } & ASSIGNIENT & \multicolumn{2}{|c|}{ FRERUENCY } & 8 & B \\
\hline & & & & & & 17( & & & & & & & $18(15,4)-17(15,3)$ & 220998.80 & & & \\
\hline & & & & 11.0 & & & & & 209801.64 & $(0.07)$ & 1.0 & & $18(14,4)-17(14,3)$ & 221049.47 & $(0.06)$ & & \\
\hline & & & $(0.04)$ & 6.0 & 124 & $17 i$ & $8,9)-16($ & $8,8)$ & 209853.60 & $(0.04)$ & 13.2 & 92 & $18(14,5)-17(14,4)$ & 221067.34 & $(0.06)$ & & \\
\hline & $3,22)-24(2.23)$ & 202094.18 & $(0.04)$ & 6.0 & 124 & $17 i$ & $8,10)-16($ & $8,9)$ & 209873.24 & $(0.04)$ & 13.2 & 92 & $29(9,21)-29(8,22)$ & 221086.08 & $(0.16)$ & 16.6 & \\
\hline & $8,12)-20(7,13)$ & 202410.34 & $(0.09)$ & 10.3 & 116 & $17 i$ & $4,14)-16($ & $4,13)$ & 209918.46 & $(0.03)$ & 16.0 & 70 & $18(13,5)-17(13,4)$ & 221139.35 & & 8.6 & \\
\hline & $8,13)-20(7,14)$ & 202571.58 & $(0.09)$ & 10.3 & 116 & & & & 210136.82 & $(0.11)$ & 4.5 & 23 & $18(13,6)-17(13,5)$ & 221158.84 & $(0.05)$ & 8.6 & \\
\hline 191 & $8,11)-19(7,12)$ & 203472.38 & $(0.06)$ & 9.5 & 108 & $17 i$ & $7,10)-16($ & 7, 9) & 210434.38 & $(0.04)$ & 14.1 & 85 & $18(12,6)-17(12,5)$ & 221260.50 & $(0.04)$ & & 136 \\
\hline 191 & $9(7,13)$ & 203500.81 & $(0.06)$ & 9.5 & 108 & $17 i$ & $7,11)-16($ & $7,10)$ & 210451.46 & $(0.04)$ & 14.1 & 85 & $18(12,7)-17(12,6)$ & 221281.09 & $(0.04)$ & 10.0 & \\
\hline 171 & $3,15)-16(3,14)$ & 203853.71 & $(0.03)$ & 16.3 & 66 & $27 i$ & $3,24)-27($ & $3,25)$ & 210541.49 & $(0.05)$ & 0.8 & 161 & $28(4,25)-28(3,26)$ & 221293.85 & $(0.07)$ & 9.0 & \\
\hline 18( & $8,11)-18(7,12)$ & 204315.54 & $(0.05)$ & & 100 & 27( & $3,24)-27($ & $2,25)$ & 210589.29 & $(0.05)$ & 9.0 & 161 & $18(11,7)-17(11,6)$ & 221424.52 & & 11.3 & 126 \\
\hline $18 i$ & $8,10)-18(7,11)$ & 204329.38 & $(0.05)$ & 8.8 & 100 & $7 i$ & $5,2)-6($ & $4,3)$ & 210725.80 & $(0.11)$ & 4.5 & 23 & $18(11,8)-17(11,7)$ & 221445.70 & $(0.04)$ & 11.3 & 126 \\
\hline 171 & $8,10)-17(7,1]$ & 205008.65 & $(0.05)$ & 8.0 & 92 & 18( & $2,16)-17($ & $3,15)$ & 210800.74 & $(0.03)$ & 9.6 & 73 & $18(10,8)-17(10,7)$ & 221649.39 & $(0.04)$ & 12.5 & \\
\hline 17( & $8,9)-17(7,10)$ & 205032.82 & $(0.05)$ & 8.0 & 92 & 23( & $1,22)-23($ & $1,23)$ & 210958.55 & $(0.07)$ & 0.3 & 108 & $18(4,15)-17(4,14)$ & 221660.46 & & & \\
\hline 301 & $7,24)-30(5,25)$ & 205038.94 & $(0.29)$ & 0.8 & 215 & $23 i$ & $1,22)-23($ & $0,23)$ & 210958.82 & $(0.07)$ & 3.0 & 108 & & & $(0.05)$ & & 144 \\
\hline 181 & $1,17)-17(2,16)$ & 205250.81 & $(0.03)$ & 13.2 & 69 & 211 & $4,17)-20($ & $5,16)$ & 211241.57 & $(0.09)$ & 4.6 & 105 & $18(10,9)-17(10,8)$ & 221670.71 & $(0.04)$ & 12.5 & 116 \\
\hline 161 & $3,14)-15(2,13)$ & 205483.24 & $(0.04)$ & 7.6 & 60 & 17( & & $6,11)$ & 211265.88 & $(0.04)$ & 14.9 & 80 & 24) $-26(2.25)$ & 221745.78 & $(0.05)$ & 6.0 & 144 \\
\hline 18( & $2,17)-17(2,16)$ & 205495.69 & $(0.03)$ & 17.6 & 69 & 27( & $4,24)-27($ & $3,25)$ & 211359.79 & $(0.05)$ & 9.0 & 161 & & 221964.54 & 10. & & \\
\hline 161 & $8,9)-16(7,10)$ & 205586.88 & $(0.05)$ & 7.3 & 85 & $17 i$ & $6,11)-16($ & $6,10)$ & 211537.44 & $(0.04)$ & 14.9 & 80 & 1819 & & & 13.5 & 108 \\
\hline $16 i$ & $8,8)-16(7,9)$ & 205613.59 & $(0.05)$ & 7.3 & 85 & $17 i$ & $5,13)-16($ & $5,12)$ & 211771.02 & $(0.03)$ & 15.5 & 75 & & 222345.08 & $(0.10)$ & 4.6 & \\
\hline 18( & $1,17)-17(1,16)$ & 205663.66 & & 17.6 & 69 & 251 & & 1,24) & 211790.75 & $(0.04)$ & 6.0 & 134 & $27(9,18)-27(8,19)$ & 222367.29 & $(0.14)$ & 15.0 & 193 \\
\hline 291 & $4,25)-29(4,26)$ & 205790.57 & $(0.08)$ & 1.0 & 190 & 301 & $9,21)-30($ & $8,22)$ & 211810.35 & $(0.24)$ & 17.5 & 229 & $18(8,10)-17($ & 222421.46 & $(0.04)$ & & \\
\hline 181 & $2,17)-17(1,16)$ & 205908.55 & $(0.03)$ & 13.2 & 69 & $25 i$ & $3,23)-25($ & $2,24)$ & 211922.83 & $(0.04)$ & 6.0 & 134 & $3(8,11)-17(8,10)$ & & & & \\
\hline 151 & $8,8)-15(7,9)$ & 206063.30 & $(0.05)$ & 6.6 & 79 & 19( & $3,16)-18($ & $4,15)$ & 212440.45 & $(0.0)-1)$ & 6.4 & 85 & $9,20)-28(8,21)$ & 222635.15 & $(0.13)$ & 15.7 & 205 \\
\hline 151 & & & & 6.6 & 79 & & & 2,14) & 212740.14 & & 8.6 & 66 & $8(5,3)-7(4,4)$ & 222925.73 & $(0.10)$ & 4.6 & 26 \\
\hline 291 & $4,25)-29(3,26)$ & 206102.54 & $(0.08)$ & 12.0 & 190 & 17( & $5,12)-16($ & $5,11)$ & 214631.75 & $(0.03)$ & 15.5 & 75 & $19(2,17)-18(3,16)$ & 223037.92 & $(0.03)$ & 10.7 & \\
\hline 161 & $4,12)-15(4,11)$ & 206247.88 & $(0.03)$ & 15.1 & 64 & 18( & $3,16)$ & $3,15)$ & 2147 & $(0.03)$ & 17.3 & 74 & $-17(7.10)$ & & & & 93 \\
\hline 191 & $0,19)-18(1,18)$ & 206364.18 & $(0.03)$ & 17.4 & 70 & $19 i$ & & $2,17)$ & 5.97 & $(0.03)$ & 14.2 & 76 & $18(7,1)$ & 223135.13 & $(0.03)$ & 15.3 & 93 \\
\hline $19 i$ & $1,19)-18(1,18)$ & 206367.38 & $(0.03)$ & 18.8 & 70 & & & $2,17)$ & & & 18.6 & 76 & $(6,13)-17(6,12)$ & 224021.67 & $(0.03)$ & 16.0 & \\
\hline 19( & $0,19)-18(0,18)$ & 206370.03 & $(0.03)$ & 18.8 & 70 & 291 & $9,20)-29($ & $8,21)$ & 216134.54 & $(0.18)$ & 16.6 & 217 & $27(9,19)-27(8,20)$ & 224167.75 & 10.100 & & 193 \\
\hline 19( & $1,19)-18(0,18)$ & 20637 & $(0.03)$ & 17.4 & 70 & 19( & 18( & $1,17)$ & & $(0.03)$ & 18.6 & 76 & $18(5,14)-17(5,13)$ & & & 16.6 & 82 \\
\hline 141 & $8,7)-14(7,8)$ & 20645 & $(0.05)$ & 5.8 & 73 & & & & & $(0.03)$ & 14.2 & 76 & $18(6,12)-17(6$ & & $(0.03)$ & 16.0 & 87 \\
\hline $14 i$ & $8,6)-14(7,7)$ & 206480.02 & $(0.05)$ & 5.8 & 73 & $18 i$ & & & 216830.11 & $(0.03)$ & 17.3 & 73 & $26(9,17)-26(8,18)$ & 224619.52 & $(0.18)$ & 14.2 & \\
\hline & $3,13)-15(3,12)$ & 206601.17 & $(0.03)$ & 15.4 & 62 & & & & 2169 & & 18.4 & 7 & -515, & 225 & $(0.14)$ & & \\
\hline 171 & $2,15)-16(2,14)$ & 20671 & $(0.03)$ & 16.4 & 66 & & 1,2 & $1,19)$ & 216 & (o. & 19.8 & 77 & $-18(3)$ & 2256 & & 18.3 & \\
\hline 131 & $8,6)-13(7,7)$ & 2067 & $(0.05)$ & 5.1 & 67 & & & $0,19)$ & & & 19.8 & 77 & $26(9,18)-26(8.19)$ & & & 14.1 & 182 \\
\hline 131 & $8,5)-$ & 206793.09 & $(0.0$ & 5.1 & 67 & 201 & $1,20)$ & $0,19)$ & 216967.98 & $(0.03)$ & 18.4 & 77 & $6(6,0)-3(5,1)$ & 226232.89 & $(0.14)$ & 5.5 & \\
\hline 121 & $8,5)-12(7,6)$ & 207011.19 & & 4.4 & 61 & & & & & $(0.09)$ & 1.0 & & $25(8,17)$ & 22646 & & & 171 \\
\hline 12( & $8,4)-12(7,5)$ & 20704 & $(0.0$ & 4.4 & 61 & & 4,26 & $3,27)$ & & $(0.09)$ & 12.0 & 20 & $19(2,18)$ & 9.40 & & 15.3 & \\
\hline & $16,1)-16(16,0)$ & & $(0.14)$ & 1.9 & 181 & & & & & & 16.4 & & $19(2,18)$ & & & 19.5 & \\
\hline & $16,2)-16(16,1)$ & 208633.81 & $(0.14)$ & 1.9 & 181 & & & $4,27)$ & 219417.33 & $(0.08)$ & 12.0 & & $1,18)$ & 226 & & 19.5 & \\
\hline $17(1$ & $15,2)-16(15,1)$ & 208659.30 & $(0.10)$ & 3.8 & 166 & 281 & $9,19)-28($ & $8,20)$ & 219592.25 & $(0.14)$ & 15.8 & & $(1,18)$ & & & & 84 \\
\hline & $15,3)-16(15,2)$ & & (0. & 3.8 & 16 & & & 8,2 & & & 17. & & $9.17)-25(8.18)$ & 226973.14 & $(0.18)$ & & \\
\hline $17(1$ & $14,3)-16(14,2)$ & 208712.80 & $(0.07)$ & & 153 & & & & & & 1.0 & & $19(2,17)-18(2,16)$ & & & 18.3 & 81 \\
\hline & $14,4)-16(14,3)$ & 208730.00 & & 5.5 & 153 & & & & 220166.85 & $(0.03)$ & 16.1 & 72 & $21(0,21)-20(1,20)$ & 227560.02 & $(0.03)$ & 19.4 & 85 \\
\hline & $13,4)-16(13,3)$ & 208786.25 & $(0.05)$ & 7.1 & 141 & & 1,23 & $1,24)$ & & & 0. & & 1,2 & & & & 85 \\
\hline & $13,5)-16(13,4)$ & & & & 14 & & & & & & & & $(0,21)-20(0,20)$ & 227561.75 & $(0.03)$ & & 85 \\
\hline & $12,5)-16(12,4)$ & 208886.17 & & 8.5 & 129 & & $3,25)-28$ & $3,26)$ & & & 0 . & 17 & $21(1.21)-20(0.20)$ & & & & \\
\hline & $12,6)-16(12,5)$ & 208905.96 & $(0.05)$ & 8.5 & 129 & 18 & $3,16)-17($ & $2,15)$ & 220811.69 & $(0.03)$ & 9.7 & 74 & & & & & \\
\hline $17(1$ & $11,6)-16(11,5)$ & 209022.27 & $(0.04)$ & & 119 & & $3.25)-28($ & $2,26)$ & & & & & $(8,1$ & 228204.82 & & & 161 \\
\hline & $11,7)-16(11$ & & & 9.9 & 11 & & $17,1)$ & 17. & & & & & $5,13)-17(5,1$ & 228628.82 & $(0.03)$ & 16.6 & 83 \\
\hline & $10,7)-16(10,6)$ & & & 11.1 & 109 & & $17,2)-17(1$ & $17,1)$ & & & & & $23(9,14)-23(8,15)$ & 229260.20 & $(0.08)$ & & \\
\hline & $10,8)-16(10,7)$ & 209230.05 & & 11.1 & 109 & $18(1$ & $16,2)-17(1$ & $16,1)$ & 220934.82 & $(0.11)$ & 3.8 & 188 & $23(9,15)-23(8,16)$ & & & 11.8 & 151 \\
\hline & $9,8)-16(9,7)$ & 209472.56 & $(0.04)$ & 12.2 & 100 & $18(1$ & $16,3)-17(1$ & $16,2)$ & 220947.86 & $(0.11)$ & 3.8 & 188 & $18(3,15)-17(3,14)$ & 229404.98 & & & 77 \\
\hline & & & & & & & & & & & & & $20(3,17)-19(4,16)$ & 229474.17 & $(0.05)$ & 7.5 & \\
\hline
\end{tabular}


TABLE 3-Continued

\begin{tabular}{|c|c|c|c|c|c|c|c|c|c|c|c|c|c|c|}
\hline ASSIGNOENT & FRBQUE & ENCY & 8 & E & ASSIGNOENT & FREQUE & ENCY & $\mathbf{s}$ & E & ASSIGNGENT & \multicolumn{2}{|c|}{ FRBQUENCY } & $\mathbf{s}$ & E \\
\hline ,17) $-18(2,16)$ & & & 10.8 & 81 & & & & & 108 & & & & & \\
\hline ,13) $-22(8,14)$ & & & & 141 & $17(17,0)-18(16,3)$ & 235050.00 & & 0.0 & & $20(11,9)-19(11,8)$ & 246285.47 & $(0.04)$ & & \\
\hline 14) $-22(8,15)$ & 230315.11 & $(0.05)$ & 11.1 & 141 & $19(8,12)-18(8,11)$ & 235051.32 & $(0.04)$ & 15.6 & 108 & $20(11,10)-19(11,9)$ & 246308.10 & $(0.04)$ & 14.0 & 142 \\
\hline $5(1,24)-25(1,25)$ & 230435.66 & $(0.08)$ & 0.3 & 127 & $9(5,4)-8(4,5)$ & 235087.58 & $(0.09)$ & 4.7 & 30 & $10(5,6)-9(4,5)$ & 246456.53 & $(0.08)$ & 4.7 & \\
\hline $5(1,24)-25(0,25)$ & 230435.73 & $(0.08)$ & 3.0 & 127 & $19(7,13)-18(7,12)$ & 235866.04 & $(0.03)$ & 16.4 & 101 & $20(10,10)-19(10,9)$ & 246600.16 & $(0.04)$ & 15.0 & 132 \\
\hline $29(3,26)-29(3,27)$ & 230920.12 & $(0.09)$ & 0.8 & 184 & $19(7,12)-18(7,11)$ & 235886.96 & $(0.03)$ & 16.4 & 101 & $20(10,11)-19(10,10)$ & 246623.01 & $(0.04)$ & 15.0 & 132 \\
\hline $29(3,26)-29(2,27)$ & 230936.36 & $(0.09)$ & 9.0 & 184 & $20(3,18)-19(3,17)$ & 236355.91 & $(0.03)$ & 19.3 & 89 & $14(7,8)-15(4,11)$ & 246674.47 & $(0.16)$ & 0.0 & \\
\hline $21(9,13)-21(8,14)$ & 231187.32 & $(0.05)$ & 10.3 & 132 & $19(5,15)-18(5,14)$ & 236743.63 & $(0.03)$ & 17.7 & 90 & $15(4,12)-14(3,11)$ & 246675.31 & $(0.06)$ & 4.5 & \\
\hline $21(9,12)-21(8,13)$ & 231200.54 & $(0.05)$ & 10.3 & 132 & $19(6,14)-18(6,13)$ & 236800.43 & $(0.03)$ & 17.1 & 95 & 5) $-18(4,14)$ & 246891.62 & $(0.03)$ & 18.2 & 88 \\
\hline $29(4,26)-29(3,27)$ & 231232.10 & $(0.09)$ & 9.0 & 184. & $21(1,20)-20(2,19)$ & 237261.25 & $(0.03)$ & 16.3 & 91 & $20(9,11)-19(9,10)$ & 247040.77 & $(0.04)$ & 16.0 & 124 \\
\hline $27(2,25)-27(1,26)$ & 231513.38 & $(0.07)$ & 6.0 & 154 & $13(4,10)-12(3,9)$ & 237296.99 & $(0.05)$ & 4.0 & 45 & $21(3,19)-20(3,18)$ & 247044.11 & $(0.03)$ & 20.3 & \\
\hline $27(3,25)-27(2,26)$ & 231559.62 & $(0.07)$ & 6.0 & 154 & $-19(2,17)$ & 237297.46 & $(0.03)$ & 19.3 & 89 & $(9,12)-19(9,11)$ & 247063.46 & $(0.04)$ & 16.0 & \\
\hline o( 8,13$)$ & 231938.57 & $(0.05)$ & 9.6 & 124 & $21(2,20)-20(2,19)$ & 237309.53 & $(0.03)$ & 20.5 & 91 & $10(5$, & & & 4.7 & \\
\hline $20(9,11)-20(8,12)$ & 231955.32 & $(0.05)$ & 9.6 & 124 & $21(1,20)-20(1,19)$ & 237344.89 & $(0.03)$ & 20.5 & 91 & $21(2,19)-20(2,18)$ & 247656.85 & $(0.03)$ & 20.3 & 97 \\
\hline $19(9,11)-19(8,12)$ & 232579.47 & $(0.04)$ & 8.9 & 115 & $21(2,20)-20(1,19)$ & 237393.17 & $(0.03)$ & 16.3 & 91 & $20(8,12)-19(8,11)$ & 247682.65 & $(0.04)$ & 16.8 & 116 \\
\hline $19(9,10)-19(8,11)$ & 232597.19 & $(0.04)$ & 8.9 & 115 & (2) $-6(5,1)$ & 237791.44 & $(0.13)$ & 5.5 & 28 & $-19(8,12)$ & 247704.35 & $(0.04)$ & 16.8 & \\
\hline $22(4,18)-21(5,17)$ & 233032.49 & $(0.09)$ & 5.5 & 115 & $19(6,13)-18(6,12)$ & 237807.69 & $(0.03)$ & 17.1 & 95 & $-21(2,20)$ & .98 & & 17.3 & \\
\hline $18(9,10)-18(8,11)$ & 233122.31 & $(0.04)$ & 8.1 & 108 & $2(0,22)-21(1,21)$ & 238155.40 & $(0.04)$ & 20.4 & 93 & $22(2,21)-21(2,20)$ & 247901.65 & $(0.03)$ & 21.5 & 100 \\
\hline $18(9,9)-18(8,10)$ & 233140.58 & $(0.04)$ & 8.1 & 108 & $(1,22)-21(1,21)$ & 238155.90 & $(0.04)$ & 21.8 & 93 & $-21(1,20)$ & 247922.26 & $(0.03)$ & 21.5 & 100 \\
\hline $19(18,1)-18(18,0)$ & 233182.54 & $(0.22)$ & 1.9 & 227 & $22(0,22)-21(0,21)$ & 238156.34 & $(0.04)$ & 21.8 & 93 & $-21(1,20)$ & & & 17.3 & \\
\hline $19(18,2)-18(18,1)$ & 233188.72 & $(0.22)$ & 1.9 & 227 & $22(1,22)-21(0,21)$ & & & 20.4 & 93 & ( 7,1 & & & 17.6 & \\
\hline $19(17,2)-18(17,1)$ & 233212 & $(0.14)$ & 3.8 & 211 & $7(6,1)-6(5,2)$ & 238488.05 & $(0.13)$ & 5.5 & 28 & ( 3,19$)-20(2,18)$ & 248673.43 & $(0.03)$ & 12.9 & 97 \\
\hline $19(4,16)-18(4,15)$ & 233212.74 & $(0.03)$ & 18.0 & 86 & $20(3,18)-19(2,17)$ & 238926.78 & $(0.03)$ & 11.8 & 89 & $20(7,13)-19(7,12)$ & 248744.68 & $(0.04)$ & 17.6 & 109 \\
\hline $19(17,3)-18(17,2)$ & 233222.42 & $(0.14)$ & 3.8 & 211 & $(3,16)-18(3,15)$ & 240021.08 & $(0.03)$ & 18.3 & 85 & $-22(1,22)$ & & & 21.4 & \\
\hline $19(16,3)-18(16,2)$ & 233255.54 & $(0.09)$ & 5.5 & 196 & $26(1,26)$ & 2401 & 0.1 & 0.3 & 136 & $22(1$, & & & 22.8 & \\
\hline $19(16,4)-18(16,3)$ & 23326 & $(0.09)$ & 5.5 & 196 & $(1,25)-26(0,26)$ & 24016 & & 3.0 & 13 & $(0,23)-22(0,22)$ & 248749.85 & $(0.04)$ & 22.8 & 101 \\
\hline $19(15,4)-18(15,3)$ & 233315.35 & $(0.06)$ & 7.2 & 182 & $(3,27)-30(3,28)$ & 240973.39 & $(0.11)$ & 0.8 & 195 & $23(1,23)-22(0,22)$ & 248750.12 & $(0.04)$ & 21.4 & 101 \\
\hline $19(15,5)-18(15,4)$ & 23333 & $(0.06)$ & 7.2 & 182 & $(3,27)-30(2,28)$ & 240982.78 & $(0.1$ & 9.0 & 19 & $(5,16)-19(5,15)$ & & & 18.7 & \\
\hline $19(14,5)-18(14,4)$ & 233396 & $(0.0$ & 8.7 & 168 & $30(3,2$ & 241163 & 10.10 & 9.0 & 19 & $(6,15)-19(6,14)$ & & & 18. & \\
\hline $19(14,6)-18(14,5)$ & 233414.76 & $(0.05)$ & 8.7 & 168 & $(2,26)-28(2,27)$ & 241334 & & 0.6 & 16 & $30(10,20)-30(9,21)$ & .45 & & 16.5 & 238 \\
\hline $19(13,6)-18(13,5)$ & 233504.71 & $(0.05)$ & 10.1 & 156 & $8(2,26)-28(1,27)$ & 241335.63 & $(0.09)$ & 6.0 & 16 & $27(1,26)-27(1,27)$ & 83.26 & $(0.15)$ & 0.3 & 146 \\
\hline $19(13,7)-18(13,6)$ & 233524.87 & $(0.0$ & 10.1 & 156 & $(3,26)-28(2,27)$ & & & 6.0 & & $27(1,26)-27(0,2$ & & & $\mathbf{3 . 0}$ & 146 \\
\hline $17(9,9)-17(8,10)$ & 2335 & 10.0 & & 100 & $28(1,2$ & 2413 & 10. & 0.6 & & 816,3 & & & 5.5 & \\
\hline $17(9,8)-17(8,9)$ & 233597.49 & $(0.04)$ & 7.4 & 100 & $9(5,14)-18(5,13)$ & 2428 & 10.0 & 17.7 & & $-19(3,16)$ & & & 19.2 & 93 \\
\hline $19(12,7)-18(12,6)$ & 233649.77 & $(0.04)$ & 11.4 & 144 & $(4,17)-19(4,16)$ & 244580.31 & $(0.03)$ & 19.1 & 9 & $16(4,13)-15(3,12)$ & 250288.70 & $(0.06)$ & 4.9 & 63 \\
\hline $19(12,8)-18(12,7)$ & 23367 & $(0.0$ & & 144 & $(3,18)-20(4,17)$ & & & & 10 & $30(9,2$ & & & 16.5 & \\
\hline $18(4,14)-17(4,13)$ & & $(0.03)$ & 17.2 & 79 & $20(19,1)-19(19,0)$ & 245466 & $(0.33)$ & 2.0 & & $8(6,2)-7(5,3)$ & 250730.69 & $(0.12)$ & 5.5 & 31 \\
\hline $26(21,5)-27(20,8)$ & 233754.58 & $(4.84)$ & 0.4 & 347 & $20(19,2)-19(19,1)$ & 245467.92 & $(0.33)$ & 2.0 & 25 & $28(6,22)-27(7,21)$ & 251081.32 & & 4.4 & 188 \\
\hline $19(11,8)-18(11,7)$ & 233845.21 & $(0.04)$ & 12.6 & 134 & $20(18,2)-19(18,1)$ & 245492.07 & & 3.8 & 23 & $29(2,27)-29(2,28)$ & & & 0.6 & 176 \\
\hline $19(11,9)-18(11,8)$ & 2338 & $(0.0$ & & 134 & $20(18,3)$ & & & & & 1,2 & & & & 176 \\
\hline & 233959.18 & $(0.04)$ & 6.7 & & $25(5,20)-24(6,19)$ & 245508.90 & $(0.17)$ & 4.8 & 14 & $29(3,27)-29(2,28)$ & 251154.49 & $(0.13)$ & & \\
\hline & 233978.53 & $(0.04)$ & 6.7 & 93 & $20(17,3)-19(17,2)$ & 245530.23 & $(0.12)$ & 5.6 & 21 & $29(3,27)-29(1,28)$ & 251154.96 & & 0. & \\
\hline $19(10,9)-18(10,8)$ & 2341 & & 13.7 & 124 & $0(17,4)-19(17,3)$ & 245540.36 & $(0.12)$ & 5.6 & 21 & $20(6,14)-19(6,13)$ & & & 18.2 & 103 \\
\hline $19(10,10)-18(10,9)$ & 234 & 10.0 & 13.7 & 124 & $16.4)$ & & & & & $(9,2)$ & .49 & & 15 & \\
\hline & 234273.25 & & & & $20(16,5)-19(16,4)$ & & & & & $-29(9.2$ & & & & 225 \\
\hline $15(9,6)-15(8,7)$ & 234293.17 & $(0.04)$ & 3.9 & 87 & $20(15,5)-19$ & 245656.57 & & 8.8 & & $28(10,18)-28(9,19)$ & & & & \\
\hline $9(5,5)-8(4,4)$ & 234479.63 & $(0.0$ & 4.7 & 30 & $20(15,6)$ & & $(0.0$ & 8.8 & & $17(4,14)-16(3,13)$ & & & 3.5 & \\
\hline & 234486.57 & 10. & 1 & 115 & $20(14,6)$ & & & & & $8(9,2$ & & & & \\
\hline 1919 & 23450 & & & 115 & $20(14,7)-19(14,6)$ & 2457 & $(0.05)$ & 10.2 & 17 & $23(4,19)-22(5,18)$ & 8.32 & $(0.09)$ & 6. & 125 \\
\hline 1419 & 234529.48 & $(0.05)$ & 5.2 & 80 & $20(13,7)-19(13,6)$ & 2458 & $(0.05)$ & 11.6 & 164 & $27(10,17)-27(9,18)$ & 254838.62 & $(0.20)$ & & \\
\hline $14(9,5)-14(8,6)$ & 234550.00 & $(0.05)$ & 5.2 & 80 & $20(13,8)-19(13,7)$ & 245903.76 & $(0.05)$ & 11. & 164 & $27(10,18)-27(9,19)$ & & $(0.20)$ & 14.2 & 202 \\
\hline $20(2,18)-19(3,17)$ & 234726.59 & $(0.03)$ & 11.8 & 89 & $21(2,19)-20(3,18)$ & & & 12 & 97 & $21(4,18)-20(4,17)$ & & & 20.1 & 102 \\
\hline $13(9,5)-13(8,6)$ & 234735 . & $(0.0$ & 4.4 & 75 & & 24605 & $(0.04)$ & 12. & & $26(10,16)-26(9,17)$ & 256072.93 & $(0.11)$ & 13.4 & 19 \\
\hline $13(9,4)-13(8,5)$ & 234756.79 & $(0.05)$ & & 75 & $25(21,5)-26(20,6)$ & 246075.92 & $(4.64)$ & 0.3 & 336 & $26(10,17)-26(9,18)$ & 256094.46 & $(0.11)$ & 13.4 & \\
\hline
\end{tabular}




\begin{tabular}{|c|c|c|c|c|c|c|c|c|c|c|c|c|c|c|c|}
\hline ASSIGNOENT & FREQUE & ENCY & $\mathbf{s}$ & $\mathbf{E}$ & ASSIGNAENI & & FREQUE & ENCY & $\mathbf{s}$ & B & ASSIGNUENT & \multicolumn{2}{|c|}{ FREQUENCY } & $\mathbf{s}$ & B \\
\hline 15$)-17(3,14)$ & & & 6.2 & 78 & & $9,12)$ & & & & & $25(1,25)-24(0,24)$ & & & & \\
\hline $22(2,20)-21(3,19)$ & 257064.43 & & 13.9 & 106 & & $9,13)$ & & & & & $22(21,2)-21(21,1)$ & 270029.99 & & 2.0 & \\
\hline $25(10,16)-25(9,17)$ & 257128.43 & & 12.7 & 180 & $21(10,11)-211$ & $9,12)$ & & & 9.7 & & $22(21,1)-21(21,0)$ & 270041.77 & $(0.93)$ & 2.0 & 307 \\
\hline $25(10,15)-25(9,16)$ & 257132.38 & $(0.08)$ & 12.7 & 180 & $21(3,18)-20($ & $3,17)$ & 260244.42 & $(0.04)$ & 20.2 & 102 & $22(20,3)-21(20,2)$ & 270052.94 & $(0.64)$ & 3.8 & 288 \\
\hline $20(5,15)-19(5,14)$ & 257226.56 & $(0.03)$ & 18.8 & 99 & $21(8,13)-20($ & $8,12)$ & 260384.19 & $(0.04)$ & 18.0 & 125 & $22(20,2)-21(20,1)$ & 270058.61 & $(0.64)$ & 3.8 & 288 \\
\hline & 257690.32 & & & 106 & $(8,14)-20($ & $8,13)$ & 260404.09 & $(0.04)$ & 18.0 & 12 & $22(19,3)-21(19,2)$ & 270086.74 & $(0.43)$ & & \\
\hline $21(20,2)-20(20,1)$ & 257748.33 & $(0.55)$ & 2.0 & 279 & $20(10,11)-20($ & $9,12)$ & 260644.13 & $(0.04)$ & 9.0 & 13 & $22(19,4)-21(19,3)$ & 270086.76 & $(0.43)$ & 5.6 & 270 \\
\hline $21(20,1)-20(20,0)$ & 257752.77 & $(0.55)$ & 2.0 & 279 & $20(10,10)-20($ & $9.11)$ & 260654.76 & $(0.04)$ & 9.0 & 132 & $22(18,4)-21(18,3)$ & 270128.36 & $(0.29)$ & 7.3 & 253 \\
\hline $21(19,2)-20(19,1)$ & 257774.08 & $(0.35)$ & 3.8 & 261 & $19(4,16)-18($ & $3,15)$ & 260793.36 & $(0.06)$ & 7.0 & 86 & $22(18,5)-21(18,4)$ & 270133.56 & $(0.29)$ & 7.3 & 253 \\
\hline $21(19,3)-20(19,2)$ & 257774.95 & $(0.35)$ & 3.8 & 261 & $30(2,28)-30($ & $2,29)$ & 260925.64 & $(0.18)$ & 0.6 & 187 & $22(3,19)-21(3,18)$ & 270174.00 & $(0.04)$ & 21.1 & 111 \\
\hline $21(18,3)-20(18,2)$ & 257807.25 & $(0.22)$ & 5.6 & 244 & $30(2,28)-30($ & $1,29)$ & 260925.89 & $(0.18)$ & 6.0 & 187 & $22(17,5)-21(17,4)$ & 270186.33 & $(0.19)$ & 8.9 & 237 \\
\hline $21(18,4)-20(18,3)$ & 257812.98 & $(0.22)$ & 5.6 & 244 & $0(3,28)-30 i$ & $2.29)$ & 260935.02 & $(0.18)$ & 6.0 & 18 & $22(17,6)-21(17,5)$ & 270196.12 & $(0.19)$ & & 237 \\
\hline $21(17,4)-20(17,3)$ & 257854.78 & $(0.14)$ & 7.2 & 228 & $(3,28)-30($ & $1,29)$ & 260935.28 & $(0.18)$ & 0.6 & 187 & $22(16,6)-21(16,5)$ & 270264.44 & $(0.13)$ & 10.4 & 222 \\
\hline $21(17,5)-20(17,4)$ & 257864.82 & $(0.14)$ & 7.2 & 228 & $19(10,10)-19$ ( & $9,11)$ & 261084.58 & $(0.04)$ & 8.2 & 124 & $22(16,7)-21(16,6)$ & 270278.16 & $(0.13)$ & 10.4 & 222 \\
\hline $21(16,5)-20(16,4)$ & 25791 & $(0.09)$ & 8.8 & 213 & & $9,10)$ & & & 8.2 & & & & & 11.8 & \\
\hline $21(16,6)-20(16,5)$ & 257933.73 & $(0.09)$ & 8.8 & 213 & $1(5,17)-20($ & $5,16)$ & 261148.87 & $(0.03)$ & 19.8 & 10 & $22(15,8)-21(15,7)$ & 270384.80 & $(0.09)$ & & 207 \\
\hline $21(15,6)-20(15,5)$ & 2580 & $(0.06)$ & 10.3 & 198 & $(7,15)$ & 7.14) & 261436.51 & $(0.04)$ & 18.7 & 11 & $22(14,8)-21(14,7)$ & 270503.43 & 10.0 & 13.1 & 194 \\
\hline $21(15,7)-20(15,6)$ & 2580 & $(0.06)$ & 10.3 & 198 & $18(10,9)$ & $9,10)$ & 261458.52 & $(0.04)$ & 7.5 & 11 & $22(14,9)-21(14,8)$ & 270523.05 & $(0.0$ & 13.1 & \\
\hline & & & & 169 & & & & & 7.5 & & & & & 14.3 & \\
\hline $24(10,14)-24(9,15)$ & 258050.08 & $(0.06)$ & 11.9 & 169 & $1(7,14)-20($ & $7,13)$ & 261715.68 & $(0.04)$ & 18.7 & 11 & $22(13,10)-21(13,9)$ & 270702.90 & $(0.06)$ & 14.3 & 182 \\
\hline $22(2,20)-21(2,19)$ & 258081.01 & $(0.03)$ & 21.3 & 106 & $17(10,8)-17($ & 9. 9) & 261773.43 & $(0.04)$ & 6.8 & 10 & $(3,17)$ & 270882.27 & $(0.06)$ & 9.0 & 102 \\
\hline $21(14,7)-20(14,6)$ & 258122.69 & $(0.05)$ & 11.7 & 185 & $17(10,7)-17 i$ & $9,8)$ & 261784.65 & $(0.04)$ & 6.8 & 109 & $22(12,10)-21(12,9)$ & 270915.97 & $(0.05)$ & 15.5 & 170 \\
\hline $21(14,8)-20(14,7)$ & & $(0.05)$ & 11.7 & 185 & $16(10,7)-16 i$ & & 262036.14 & & 6.0 & 10 & & 270938.92 & & & \\
\hline $11(5,7)-10(4,6)$ & 25815 & $(0.07)$ & 4.8 & 39 & $16(10,6)-16 i$ & 9. 7) & 262047.64 & $(0.04)$ & 6.0 & 10 & $22(11,11)-21(11,10)$ & 271229.32 & $(0.05)$ & 16.5 & 160 \\
\hline $21(13,8)-20(13,7)$ & 25827 & $(0.05)$ & 13.0 & 173 & $15(10,6)-15 i$ & $9,7)$ & 262252.93 & $(0.04)$ & 5.3 & 9 & $2(11,12)-21(11,11)$ & 271253.08 & 10.0 & 16.5 & 160 \\
\hline $21(13,9)-20(13,8)$ & 25829 & $(0.05)$ & 13.0 & 173 & $15(10,5)-15 i$ & $9,6)$ & 262264.71 & $(0.04)$ & 5.3 & & $-20(5,15)$ & 271505.89 & $(0.0)$ & 19. & 108 \\
\hline & & & & 108 & $9(6,4)-8($ & & & & 3.6 & & $21(4,17)-20(4,16)$ & & & & \\
\hline $21(12,9)-20(12,8)$ & 258476.61 & $(0.05)$ & 14.2 & 161 & $21(6,16)-20($ & $6,15)$ & 262324.81 & $(0.03)$ & 19.3 & 112 & $22(10,12)-21(10,11)$ & 271656.11 & $(0.05)$ & 17.5 & 150 \\
\hline $23(2,22)-22(2,21)$ & 258490.87 & $(0.04)$ & 22.5 & 108 & $(10,5)-14)$ & $9,6)$ & 262429.57 & $(0.04)$ & 4.5 & 89 & $22(10,13)-21(10,12)$ & 271680.22 & $(0.05)$ & 17.5 & 150 \\
\hline $21(12$, & 1 & $(0.0$ & 14.2 & 161 & $14(10,4)$ & $9,5)$ & & & 4.5 & & $(4,8)$ & & & 4.8 & \\
\hline $23(1,22)-22(1,21)$ & & $(0.04)$ & 22.5 & 108 & $9(6,3)-8($ & $5,4)$ & & $(0.1$ & 5.6 & 3 & $22(9,13)-21(9,12)$ & & & 18.3 & 141 \\
\hline $23(2,22)-22(1,21)$ & 258518.54 & $(0.04)$ & 18.3 & 108 & $21(6,15)-20($ & $6,14)$ & 265002.63 & $(0.03)$ & 19.3 & 112 & $22(9,14)-21(9,13)$ & 272279 & $(0.04)$ & 18.3 & 141 \\
\hline & & & & 10 & & & & & 8.0 & 9 & $-22(4.19)$ & & & & \\
\hline $21(1$ & & & & 151 & & & & & 6.5 & & $23(5$, & & & 7. & \\
\hline 1) $-20(11,10)$ & 2587 & $(0.05)$ & 15.3 & 151 & & & & $(0.18)$ & 6.5 & 34 & $22(5,18)-21(5,1$ & 78.64 & $(0.03)$ & 20.8 & \\
\hline & 2588 & $(0.06)$ & & 15 & & $4,18)$ & & & 21.1 & 11 & $22(8,14)-21(8,1$ & & & 19.1 & 134 \\
\hline$-23(9,14)$ & 2588 & 10. & & 15 & & & & $(0.04)$ & 15.0 & 11 & $-21(8,1$ & & $(0.0)-10$ & 19.1 & 134 \\
\hline $21(10,1$ & & & & 141 & & & & & & & $21(7$. & & & 19.8 & 127 \\
\hline $21(10,12)-20(10,11$ & 2591 & 10.0 & 16.3 & 141 & $(2,21$ & & 268552.69 & 10.0 & 22.3 & 11 & $10<6$ & & & 5.6 & 39 \\
\hline & & & & 11 & & & & & & & & & & & 127 \\
\hline 1,24$)-23(1,2$ & 259 & 10.0 & 23.8 & 11 & 1,2 & $2,22)$ & & 0.0 & 19.3 & 11 & $(6,16)$ & & & 20.4 & 121 \\
\hline 24) $-23(0,2$ & & & & 110 & & & & & & & & & & 5.6 & 39 \\
\hline 24) $-23(0,23)$ & 259342.33 & $(0.05)$ & 22.4 & 110 & $(1,23)-23)$ & $1,22)$ & 84.88 & $(0.0)-100$ & 23.5 & 11 & $29(6,23)-28(7,22)$ & 277410.37 & $(0.37)$ & 5.0 & \\
\hline $11(5,6)-10(4,7)$ & 259444.96 & $(0.07)$ & 4.8 & 39 & $(2,23)-23 i$ & 1,22) & 269093.79 & $(0.04)$ & 19.3 & 11 & $22(4,19)-21(3,18)$ & & & 10.1 & 111 \\
\hline $20(4.16)-19(4.15)$ & 259499.92 & $(0.03)$ & 19.2 & 96 & & $0,29)$ & 269307.22 & & & & $8(7,2)-7(6,1)$ & & & 6.5 & 37 \\
\hline & & & & & & & & & & & $23(4,20)-22(4,19)$ & & & 22.1 & 121 \\
\hline $22(10,13)-22(9,14)$ & 259530.10 & $(0.05)$ & 10.4 & 150 & $29(2,28)-291$ & 0,291 & 269307.69 & $(0.27)$ & 0.0 & 16 & $8(7,1)-7(6,2)$ & 278464.57 & $(0.17)$ & 6.5 & \\
\hline $1(4,18)$ & .16 & $(0.0$ & 9. & 111 & $29(2,28)-$ & $1,29)$ & & & 3.0 & & $24(2,22)-23(3,21)$ & & & 16.0 & 124 \\
\hline . & .62 & $(0.0)-1)$ & 10. & 15 & - & $4,8)$ & & & 0.0 & & $24(3,22)-23(3,21)$ & & & 23.3 & \\
\hline $28(1,27)-28($ & 2595 & & & 157 & & & & & 4.8 & & $1,29)-30(0,30)$ & 279011.30 & & 3. & 179 \\
\hline $28(1,27)-28(0,28)$ & 259597.98 & $(0.20)$ & 3.0 & 157 & $26(5,21)-25 i$ & $6,20)$ & 269525.60 & $(0.19)$ & 5.6 & 160 & $30(1,29)-30(1,30)$ & 279011.31 & & & \\
\hline $28(2,27)-28(1,28)$ & 259598.84 & $(0.20)$ & 3. & 157 & $25(0,25)-$ & 1,24) & 269933.13 & & & 119 & $30(2,29)-30(0,30)$ & & & & 179 \\
\hline $28(2,27)-28(0,28)$ & 259598.84 & $(0.20)$ & 0.0 & 157 & & & & & & & $30(2,29)-30(1,30)$ & 279011.56 & & & \\
\hline $21(9,12)-20(9,11)$ & 259629.42 & $(0.04)$ & 17.2 & 132 & $25(0,25)-24($ & $0.24)$ & 269933.27 & $(0.06)$ & 24.8 & 119 & $22(6,16)-21(6,15)$ & 279050.57 & $(0.03)$ & 20.4 & \\
\hline
\end{tabular}


TABLE 3-Continued

\begin{tabular}{|c|c|c|c|c|c|c|c|c|c|c|c|c|c|c|}
\hline \multirow{2}{*}{$\begin{array}{l}\text { ASSIGNUENT } \\
(2,22)-23(2,21)\end{array}$} & \multicolumn{2}{|c|}{ FREQUENCY } & \multirow{2}{*}{$\frac{S}{23.3}$} & \multirow{2}{*}{$\frac{\text { B }}{124}$} & ASSIGNMENT & \multicolumn{2}{|c|}{ FREQUENCY } & $\mathbf{s}$ & E & ASSIGNMENT & \multicolumn{2}{|c|}{ FREQUENCY } & \multicolumn{2}{|l|}{$\mathbf{s}$} \\
\hline & & & & & $27(11,17)-27(10,18)$ & 284879.53 & & & & & & & & \\
\hline & 279287.07 & & 16.0 & & $27(11,16)-27(10,17)$ & 284887.26 & $(0.13)$ & 13.5 & 211 & $26(2,25)-25(1,24)$ & 290252.80 & $(0.06)$ & 21.3 & \\
\hline $25(1,24)-24(2,23)$ & & & & 127 & $23(9,14)-22(9,13)$ & 284920.35 & $(0.05)$ & 19.5 & 151 & $25(4,21)-24(5,20)$ & 290695.42 & $(0.09)$ & 8.9 & \\
\hline & 279663.60 & $\begin{array}{l}(0.05) \\
(0.05)\end{array}$ & 24 & 127 & $23(9,15)-22(9,14)$ & 284944.89 & $(0.05)$ & 19.5 & 151 & $9(7,2)-8(6,3)$ & 290711.20 & $(0.16)$ & 6.5 & 41 \\
\hline $\begin{array}{l}5(1,24)-24(1,23) \\
5(2,24)-24((1,23)\end{array}$ & $\begin{array}{l}279667.50 \\
279672.52\end{array}$ & $\left.\begin{array}{l}(0.05) \\
(0.05)\end{array}\right)$ & $\begin{array}{l}24.5 \\
20.3\end{array}$ & $\begin{array}{l}127 \\
127\end{array}$ & $23(4,20)-22(3,19)$ & 285016.22 & $(0.05)$ & 11.2 & & $27(0,27)-26(1,26)$ & 291111.29 & $(0.09)$ & 25.4 & \\
\hline & 279975.33 & $\begin{array}{l}(0.05) \\
(0.05)\end{array}$ & $\begin{array}{r}20.3 \\
4.8\end{array}$ & $\begin{array}{r}127 \\
49\end{array}$ & $24(3,21)-23(4,20)$ & 285351.75 & $(0.05)$ & 12.3 & 130 & $27(1,27)-26(1,26)$ & 291111.30 & $(0.09)$ & 26.8 & \\
\hline & 280147.66 & $(0.04)$ & 22.1 & 120 & $22(5,17)-21(5,16)$ & 285515.70 & $(0.04)$ & 21.0 & 118 & $27(0,27)-26(0,26)$ & 291111.32 & $(0.09)$ & 26.8 & \\
\hline & 280147.00 & $(0.04)$ & 22.1 & 120 & $26(11,16)-26(10,17)$ & 285713.45 & $(0.10)$ & 12.7 & 200 & $27(1,27)-26(0,26)$ & 291111.34 & $(0.09)$ & 25.4 & \\
\hline $\begin{array}{l}60,26)-25(1,25) \\
6(1,26)-25(1,25)\end{array}$ & $\begin{array}{l}280522.93 \\
280522.97\end{array}$ & $\left.\begin{array}{l}(0.88) \\
(0.08)\end{array}\right)$ & $\begin{array}{l}24.4 \\
25.8\end{array}$ & $\begin{array}{l}128 \\
128 .\end{array}$ & $26(11,15)-26(10,16)$ & 285720.60 & $(0.10)$ & 12.7 & 200 & $27(5,22)-26(6,21)$ & 292558.76 & $(0.21)$ & 6.6 & 172 \\
\hline & $\begin{array}{l}280522.97 \\
280523.00\end{array}$ & $(0.08)$ & $\begin{array}{l}25.8 \\
25.8\end{array}$ & 128 & $23(8,16)-22(8,14)$ & 285736.70 & $(0.28)$ & 0.8 & 143 & $23(6,17)-22(6,16)$ & 293388.53 & $(0.04)$ & 21.5 & \\
\hline & 280523.04 & & 25.8 & $\begin{array}{l}128 \\
128\end{array}$ & $23(8,16)-22(8,15)$ & 285940.81 & $(0.05)$ & 20.2 & 143 & $24(4,21)-23(3,20)$ & 293410.04 & $(0.05)$ & 12.3 & \\
\hline $6(1,26)-25(0,25)$ & 281624.31 & & 24.4 & 12 & $23(8,15)-22(8,14)$ & 285973.15 & $(0.05)$ & 20.2 & 143 & $23(4,19)-22(4,18)$ & 293764.48 & & 22.1 & \\
\hline $30(11,20)-30(10,20)$ & $\begin{array}{l}281624.31 \\
281645.90\end{array}$ & $(0.44)$ & $\begin{array}{r}5.3 \\
15.7\end{array}$ & 247 & $23(8,15)-22(8,15)$ & 286089.82 & $(0.28)$ & 0.8 & 143 & $24(22,3)-23(22,2)$ & 294612.32 & & 3.8 & \\
\hline & $\begin{array}{l}281645.90 \\
281710.87\end{array}$ & $(0.40)$ & 15.7 & 247 & $25(11,15)-25(10,16)$ & 286448.89 & & 12.0 & 18 & $24(22,2)-23(22,1)$ & 294636.52 & $(1.80)$ & 3.8 & \\
\hline $\begin{array}{l}30(11,20)-30(10,21) \\
30(11,19)-30(10,21)\end{array}$ & $\begin{array}{l}281710.87 \\
281813.31\end{array}$ & $(0.40)$ & 15.7 & $\begin{array}{l}247 \\
247\end{array}$ & $25(11,14)-25(10,15)$ & 286455.22 & $(0.08)$ & 12.0 & 189 & $24(21,4)-23(21,3)$ & 294637.61 & $(1.34)$ & 5.6 & 26 \\
\hline & 28101 & $(0.44)$ & 5.3 & 247 & $11(6,6)-10(5,5)$ & 286655.61 & $(0.11)$ & 5.7 & 44 & $24(21,3)-23(21,2)$ & & & & \\
\hline $23(22,2)-22(2$ & 282312.94 & & & 336 & $24(11,14)-24(10,15)$ & 287095.15 & $(0.07)$ & 11.3 & 179 & $24(20,5)-23(20,4)$ & 294674.64 & & 7.3 & \\
\hline $23(21,3)-22($ & 282332.07 & (1.11) & 3.8 & 316 & $24(11,13)-24(10,14)$ & & & & 17 & $24(20,4)-23(20,3)$ & 294684.04 & $(0.98)$ & 7.3 & 7 \\
\hline $23(22,1)-22(22,0)$ & 282333.76 & (1.52) & 2.0 & 336 & $23(7,17)-22(7,16)$ & 287146.45 & $(0.04)$ & 20.9 & 137 & $24(19,6)-23(19,5)$ & 294725.46 & $(0.72)$ & 9.0 & 39 \\
\hline $23(21,2)-22(21,1)$ & 282345.96 & (1.11) & 3.8 & 316 & $11(6,5)-10(5,6)$ & 287281.13 & $(0.11)$ & 5.7 & 44 & $24(19,5)-23(19,4)$ & & & & \\
\hline $23(20,4)-22(20,3)$ & 282361.67 & $(0.79)$ & 5.6 & 297 & $23(6,18)-22(6,17)$ & 287592.80 & $(0.03)$ & 21.4 & 131 & $24(18,6)-23(18,5)$ & 294789.58 & $(0.52)$ & 10.5 & \\
\hline $23(20,3)-22(20,2)$ & 282368.95 & $(0.79)$ & 5.6 & 297 & $23(11,13)-23(10,14)$ & 287660.79 & & & & $24(18,7)-23(18,6)$ & 294792.82 & $(0.52)$ & 10.5 & 272 \\
\hline $23(19,5)-22(19,4)$ & 282403.54 & $(0.56)$ & 7.3 & 279 & $23(11,12)-23(10,13)$ & 287665.86 & $(0.06)$ & 10.5 & 16 & $24(17,7)-23(17,6)$ & 1.86 & $(0.37)$ & 12.0 & \\
\hline $23(19,4)-22(19,3)$ & 282404.69 & $(0.56)$ & 7.3 & 279 & $23(7,16)-22(7,15)$ & 288083.88 & $(0.04)$ & 20.9 & 137 & $24(17,8)-23(17,7)$ & & & 12.0 & \\
\hline $23(18,5)-22(18,4)$ & 282455.70 & $(0.39)$ & 8.9 & 262 & $22(11,12)-22(10,13)$ & 288153.61 & $(0.06)$ & 9.8 & 160 & $24(16,8)-23(16,7)$ & $2949^{\circ}$ & $(0.26)$ & 13.3 & \\
\hline $23(18,6)-22(18,5)$ & 282460.10 & $(0.39)$ & 8.9 & 262 & $22(11,11)-22(10,12)$ & 288158.24 & & 9.8 & 160 & $24(16,9)-23(16,8)$ & 294992.97 & $(0.26)$ & 13.3 & 241 \\
\hline $23(17,6)-22(17,5)$ & 282525.24 & $\begin{array}{l}(0.27) \\
(0.27)\end{array}$ & 10.4 & 246 & $24(4,21)-23(4,20)$ & 288541.48 & $(0.04)$ & 23.1 & & $24(15,9)-23(15,8)$ & 59 & & & \\
\hline $23(17,7)-22(17,6)$ & 282534.53 & $(0.27)$ & 10.4 & 246 & $21(11,11)-21(10,12)$ & 288580.75 & $(0.05)$ & 9.1 & 151 & $24(15,10)-23(15,9)$ & & & 14.6 & \\
\hline $23(16,7)-22(16,6)$ & 282617.64 & $(0.19)$ & 11.9 & 231 & $1,10)-21(10,11)$ & 288585.04 & $(0.0$ & 9.1 & 151 & $24(14,10)-23(14,9)$ & & $(0.13)$ & 15.8 & \\
\hline $23(16,8)-22(16,7)$ & 282631.12 & $(0.19)$ & 11.9 & 23 & $20(11,10)-20(1$ & 288948.76 & $(0.0$ & 8.3 & & $24(14,11)-23(14,10)$ & 295322.59 & $(0.13)$ & 15.8 & 213 \\
\hline $23(15,8)-22(15,7)$ & 282738.75 & & 13.2 & 21 & $20(11,9)-20(10,10)$ & 288952.77 & $(0.05)$ & 8.3 & 14 & $24(13,11)-23(13,10)$ & & $(0.10)$ & 17.0 & \\
\hline $23(15,9)-22(15,8)$ & 282755.71 & $(0.13)$ & 13.2 & 217 & $19(11,9)-19(10,10)$ & 289263.66 & $(0.05)$ & 7.6 & 134 & $24(13,12)-23(13,11)$ & 29556 & $(0.10)$ & 17.0 & \\
\hline $29(11,18)-29(10,19)$ & 282871.43 & $(0.25)$ & 15.0 & 23 & $19(11,8)-19(10,9)$ & 289267.47 & $(0.0$ & 7. & 134 & $24(12,12)-23(12,11)$ & 851.25 & $(0.08)$ & 18.0 & \\
\hline $29(11,19)-29(10,20)$ & 282882 . & $(0.25)$ & & 235 & $25(2,23)-24(3,22)$ & 289355.15 & & 17. & 134 & $24(12,13)-23(12,12)$ & 295874.76 & $(0.08)$ & 18.0 & 189 \\
\hline 9) $-22(14,8)$ & $\begin{array}{l}282896.64 \\
282916.37\end{array}$ & $\begin{array}{l}(0.10) \\
(0.10)\end{array}$ & $\begin{array}{l}14.5 \\
14.5\end{array}$ & $\begin{array}{l}203 \\
203\end{array}$ & $25(3,23)-24(3,22)$ & 289492.25 & $(0.05)$ & 24. & 13 & $24(11,13)-23(11,12)$ & 296265.81 & $(0.07)$ & 19.0 & \\
\hline $2(14,9)$ & 282939.78 & $(0.04)$ & 21.2 & 115 & $18(11,8)-18(10,9)$ & 289531.04 & $(0.05)$ & 6.8 & 12 & $24(11,14)-23(11,13)$ & 296290.33 & $(0.07)$ & 19.0 & \\
\hline $22(4,18)-$ & 283102.76 & $(0.08)$ & 15.7 & 19] & $8(11,7)-18(10,8)$ & 289534.67 & $(0.05)$ & 6.8 & & $24(5,20)-23(5,19)$ & 296342.96 & 0.04 & 22.8 & \\
\hline $\begin{array}{l}\text { 10) }-22(13,9) \\
1)-23(20,4)\end{array}$ & 283123.80 & & & 30 & $25(2,23)-24(2,22)$ & 289584.71 & $(0.05)$ & 24.3 & 134 & $24(10,14)-23(10,13)$ & 296830.89 & $(0.06)$ & 19.8 & 16 \\
\hline $\begin{array}{l}22(21,1)-23(20,4) \\
23(13,11)-22(13,10)\end{array}$ & $\begin{array}{l}283123.80 \\
283124.59\end{array}$ & $\begin{array}{l}(4.61) \\
(0.08)\end{array}$ & $\begin{array}{l}0.1 \\
15.7\end{array}$ & $\begin{array}{l}307 \\
191\end{array}$ & $14(5,10)-13(4,9)$ & 289591.73 & $(0.04)$ & 4.8 & 55 & $24(10,15)-23(10,14)$ & 296855.97 & $(0.06)$ & 19.8 & \\
\hline $23(12,11)-22(12,1$ & 283373.86 & $(0.06)$ & 16.8 & 180 & $25(3,23)-24(2,22)$ & 289721.81 & $(0$. & 17.0 & & $25(3,22)-24(4,21)$ & 297213.44 & $(0.06)$ & 13.4 & \\
\hline & & & 16.8 & 18 & $17(11,7)-17(10,8)$ & & & & & & & & & \\
\hline $23(11,12)-22(11,11)$ & 2837 & $(0.06)$ & 17.8 & 16 & $\begin{array}{l}17(11,6)-17(10,7) \\
16(11,6)-16(10,7)\end{array}$ & 289759.55 & $(0.05)$ & & 119 & $24(9,15)-23(9,14)$ & 297628.81 & $(0.05)$ & 20.6 & 161 \\
\hline $23(11,13)-22(11,12)$ & 283759.53 & $(0.06)$ & 17.8 & 169 & $\begin{array}{l}16(11,6)-16(10,7) \\
16(11,5)-16(10,6)\end{array}$ & $\begin{array}{l}289943.48 \\
289946.87\end{array}$ & $\begin{array}{l}(0.05) \\
(0.05)\end{array}-1$ & 5.3 & 112 & $24(9,16)-23(9,15)$ & 297652.97 & $(0.05)$ & 20.6 & \\
\hline & 283937.70 & & & 22 & $9(7,3)-8(6,2)$ & $\begin{array}{l}0.01 \\
7.07\end{array}$ & & 6.5 & 4 & & & & 4.8 & \\
\hline & & & & & $5(10.6)$ & & & & & $12(6,7)-11(5,6)$ & & & & \\
\hline $23(10,13)-22(10$ & & & & & $15(11,5)-15(10,6)$ & 290 & & & & $24(8,17)-23(8,16)$ & 298767.33 & $(0.07)$ & & \\
\hline $23(10,14)-22(10,13)$ & 284252.35 & $(0.05)$ & 18.7 & 159 & $24(3,21)-23(3,20)$ & 290220 & $(0.05)$ & & 13 & $24(8,16)-23(8,15)$ & 298893.46 & $(0.07)$ & 21.3 & \\
\hline & 284398.17 & & 4.8 & 49 & & & 政 & 0 & 13 & $\begin{array}{l}23(5,18)-22(5,17) \\
25(4,22)-24(4,21)\end{array}$ & 299097.82 & & 22.0 & \\
\hline $23(5,19)-22(5,18)$ & 34810.29 & & & & $26(2,25)-25(2,24)$ & 290247.78 & & & 136 & $\begin{array}{l}25(4,2)-24(4,21) \\
12(6,6)-11(5,7)\end{array}$ & $\begin{array}{l}299268.23 \\
299366.07\end{array}$ & $\begin{array}{l}(0.05) \\
(0.11)\end{array}$ & $\begin{array}{r}24.1 \\
5.8\end{array}$ & \\
\hline
\end{tabular}

NoTE. - Transition frequencies in MHz. Energies $(E)$ in $\mathrm{cm}^{-1}$. Strengths $(S)$ given are the standard line strength parameters (Townes and Schawlow 1955). 
where the torsion-rotation term $\boldsymbol{H}_{\mathrm{tr}}$ is given by

$$
\boldsymbol{H}_{\mathrm{tr}}=F \sum_{k=0}^{9} W_{v \boldsymbol{\sigma}}^{(k)}(\boldsymbol{\rho} \cdot \boldsymbol{P})^{k}
$$

The first two terms of equation (1) are the standard rotationcentrifugal distortion $\boldsymbol{A}$-reduced Hamiltonian of Watson (1968), plus a term in $\boldsymbol{P}^{2}$ (Gordy and Cook 1984). The remaining term is written in terms of the angular-momentum operator $\boldsymbol{P}$ and the vector $\boldsymbol{\rho}$, the components of which are given by

$$
\rho_{g}=\lambda_{g} I_{\alpha} / I_{g}
$$

Here the $\lambda_{g}$ are the direction cosines of the methyl group symmetry axis in the body-fixed principal-axis system, $I_{\alpha}$ is the methyl group moment of inertia about its symmetry axis, the $I_{g}$ are the principal moments of inertia of the entire molecule, and $F$ is the reduced rotational constant for the methyl group (Gordy and Cook 1984). In a prolate planar case such as methyl formate, $\lambda_{c}$ vanishes.

The advantage of expressing the torsion-rotation interaction as in equation (2) is as follows: $\boldsymbol{H}_{\mathrm{tr}}$ contains information about the asymmetry of the molecule only in the matrix elements of $\boldsymbol{\rho} \cdot \boldsymbol{P}$, whereas the dimensionless coefficients $W_{v \boldsymbol{\sigma}}^{(k)}$ depend only on the reduced barrier height $s$ (Gordy and Cook 1984). The parameter $s$ is defined for a threefold torsional barrier in terms of $F$ and the barrier height $V_{3}$ as

$$
s=(4 / 9)\left(V_{3} / F\right) \text {. }
$$

As realized by Herschbach (1959), this allows evaluation of the $W_{v \sigma}^{(k)}$ in the symmetric top limit, a fact which greatly simplifies the calculation. We have determined the $W_{v \sigma}^{(k)}$ using the internal axis method (IAM) program of Herbst (Herbst et al. 1984). The relation between the PAM coefficients and the IAM torsional eigenvalues in the symmetric top limit is given by Herschbach (1959).

Consideration of the torsional wave function symmetry leads to the fact that the $W_{v \sigma}^{(k)}$ vanish for odd-order $k$ in the $A$ state, and are of opposite sign for the two $E$ states (Herschbach 1959). Because of the selection rule $\Delta \sigma=0$, and since the even $k$ terms in $\boldsymbol{H}_{\mathrm{tr}}$ exhibit the same dependence on the $\boldsymbol{P}_{g}$ as the terms in $\boldsymbol{H}_{\text {dist }}$, analysis of the $A$ state can be carried out without explicit mention of $\boldsymbol{H}_{\mathrm{tr}}$. The "centrifugal distortion" constants obtained in the fit are, of course, actually linear combinations of the real distortion constants and the even-order $\boldsymbol{H}_{\mathrm{tr}}$ constants. The favorable results of our analysis of the $A$ state according to this approach have shown that the Van Vleck transformation method is reasonable in the case of methyl formate.

However, our determination of the rotational energies for
However, our determination of the rotational energies for the $E$ states, in which the odd-order $k$ terms of $\boldsymbol{H}_{\mathrm{tr}}$ are present, employs further approximations. We have chosen to treat this term by retaining only those matrix elements of $\boldsymbol{H}_{\mathrm{tr}}$ which connect $K$-doublet states (pairs of states corresponding to the same $|K|$ in the prolate limit). After diagonalization of the matrix $\boldsymbol{H}_{\text {rot }}+\boldsymbol{H}_{\text {dist }}$, these matrix elements are folded onto the diagonal with a series of $2 \times 2$ diagonalizations. An additional approximation used in our treatment is that the matrix elements of $\boldsymbol{H}_{\mathrm{tr}}$ are evaluated in the symmetric top basis set rather than in the pseudorigid asymmetric rotor basis set. These matrix elements then appear as simple functions of $J, K$, and the parameters $\rho_{g}$ (Herschbach 1959).

We have found that allowances for centrifugal distortion of the methyl group rotational constant $F$ and the direction cosines $\lambda_{g}$ enable us to make a more reliable set of frequency predictions; in the fitting routine, the independent parameters $F$ and $\rho_{a}$ are replaced by the forms

$$
\begin{aligned}
& F^{\prime}=F+\delta_{F} J(J+1) K^{2}, \\
& \rho_{a}^{\prime}=\rho_{a}+\delta_{\rho} K^{2} .
\end{aligned}
$$

The rotational constants $A, B$, and $C$ and 12 centrifugal distortion constants describe the unperturbed pseudorigid rotor energies, and five free parameters $\left(F, s, \rho_{a}, \delta_{F}\right.$, and $\left.\delta_{\rho}\right)$ determine the shifts in these levels due to internal rotation. Results of the analysis and the derived rotational parameters are listed in Table 2.

\section{DISCUSSION}

Table 3 lists the $\sim 1300$ strongest transitions of methyl formate from 1 to $300 \mathrm{GHz}$ in its ground torsional $E$ substate with $J \leq 30$ and $E_{\text {rot }} \leq 350 \mathrm{~cm}^{-1}$. The line strengths $S$ were calculated according to the definition of Townes and Schawlow (1955). To account for strong features due to the coincidence of two or more lines, "blend" strengths were calculated for each line by adding the quantity $\mu^{2} S$ for lines within 1.5 $\mathrm{MHz}$ of one another. Here $\mu$ represents the appropriate dipole moment component (Bauder 1979). A lower limit of 2.0 was placed on the blend strengths; this limit corresponds to lines approximately 3 times weaker than the weakest lines assigned (at $E / k \sim 2 T_{\text {Orion }}$ ) by Sutton et al. (1985) and Blake et al. (1986) in their Orion line search. The selection criteria match those of our previous listing of the spectrum of the $A$ species.

The table includes the assignments, frequencies, uncertainties in the frequencies ( $1 \sigma)$, line strengths $S$, and excitation energies for the selected transitions. We note that as a result of mixing of the asymmetric top $K$-doublet wave functions, several unallowed asymmetric rotor transitions appear.

We acknowledge the support of the National Aeronautics and Space Administration via grant NAGW-189.

\section{REFERENCES}

Bauder, A. 1979, J. Phys. Chem. Ref. Data, 8, 583.

Blake, G. A., Sutton, E. C., Masson, C., and Phillips, T. G. 1986, Ap. J. Suppl. 60, 357.

Curl, R. F. 1959, J. Chem. Phys., 30, 1529.

De Lucia, F. C. 1976, in Molecular Spectroscopy, Modern Research, ed. K. N. Rao (New York: Academic), p. 69.
DeMaison, J., Boucher, D., Dubrille, A., and Van Eijck, B. P. 1983, J. Molec. Spectrosc., 120, 260.

Gordy, W., and Cook, R. L. 1984, Microwave Molecular Spectra (New York: Wiley)

Helminger, P., Messer, J. K., and De Lucia, F. C. 1983, Appl. Phys. Letters, 42, 309. 
Herbst, E, Messer, J. K., De Lucia, F. C., and Helminger, P. 1984, J. Molec. Spectrosc., 108, 42

Herschbach, D. R. 1957, Tables for the Internal Rotational Problem (Cambridge: Harvard University Press)

1959, J. Chem. Phys., 31, 91.

Johansson, L. E. B., et al. 1984, Astr. Ap., 130, 227.

IOI Lovas, F. J., Lutz, H., and Dreizler, H. 1979, J. Phys. Chem. Ref. Data,

II. 8, 1051

Geoffrey A. Blake: Department of Chemistry, University of California, Berkeley, CA 94720

Frank C. De Lucia and Eric Herbst: Department of Physics, Duke University, Durham, NC 27706

Grant M. Plummer: I. Physikalisches Institut der Universität zu Köln, Cologne, West Germany York: McGraw-Hill).
Plummer, G. M., Blake, G. A., Herbst, E., and De Lucia, F. C. 1984, Ap. J. Suppl., 55, 633.

Sutton, E. C., Blake, G. A., Masson, C., and Phillips, T. G. 1985, Ap. J. Suppl., 58, 341 .

Townes, C. H., and Schawlow, A. L. 1955, Microwave Spectroscopy (New

Watson, J. K. G. 1968, J. Chem. Phys., 48, 4517. 